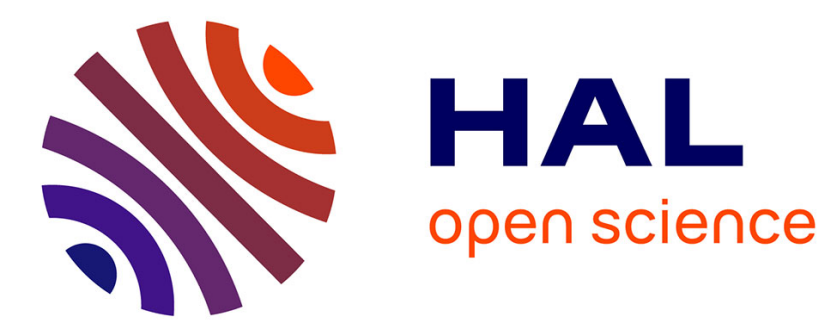

\title{
Error distribution in randomly perturbed orbits
}

Sandro Vaienti, Giorgio Turchetti, Francesco Zanlungo, Philippe Marie

\section{To cite this version:}

Sandro Vaienti, Giorgio Turchetti, Francesco Zanlungo, Philippe Marie. Error distribution in randomly perturbed orbits. Chaos: An Interdisciplinary Journal of Nonlinear Science, 2009, 19, pp.043118 (2009). 10.1063/1.3267510. hal-00476224

\section{HAL Id: hal-00476224 \\ https://hal.science/hal-00476224}

Submitted on 24 Apr 2010

HAL is a multi-disciplinary open access archive for the deposit and dissemination of scientific research documents, whether they are published or not. The documents may come from teaching and research institutions in France or abroad, or from public or private research centers.
L'archive ouverte pluridisciplinaire HAL, est destinée au dépôt et à la diffusion de documents scientifiques de niveau recherche, publiés ou non, émanant des établissements d'enseignement et de recherche français ou étrangers, des laboratoires publics ou privés. 


\title{
1 Error distribution in randomly perturbed orbits
}

\author{
Ph. Marie, ${ }^{1, \text { a) }}$ G. Turchetti, ${ }^{2, b)}$ S. Vaienti, ${ }^{1,3, c)}$ and F. Zanlungo ${ }^{2, d)}$ \\ ${ }^{1}$ Centre de Physique Théorique, CNRS, UMR-6207, Universités d'Aix-Marseille I, II, \\ Provence-Alpes-Côte-d'Azur 13284, France and Université du Sud, Toulon-Var, Toulon 83130, France \\ ${ }^{2}$ Department of Physics and Centro Galvani, University of Bologna, Bologna 40126, Italy \\ ${ }^{3}$ Fédération de Recherche des Unités de Mathématiques de Marseille (FRUMAM), CPT, \\ Luminy Case 907, F-13288 Marseille Cedex 9, France
}

$8 \quad$ (Received 18 May 2009; accepted 3 November 2009; published online xx xx xxxx)

21 In the present paper we study the effect of a random 22 perturbation on the orbit of a discrete dynamical system. 23 We analyze the statistics of global errors $\Delta_{n}^{\varepsilon}$, i.e., of the 24 algebraic difference at iteration $\boldsymbol{n}$ between the exact orbit 25 and an orbit perturbed at each step with a random error 26 of order $\varepsilon$, by providing exact results for two model 27 maps, regular and chaotic, respectively, and stating a 28 general theorem on their asymptotics. This analysis sug29 gests the existence of a time scale depending on $\varepsilon$ for the 30 convergence to the asymptotic error distribution. The 31 scale is basically $\log (1 / \varepsilon)$ for chaotic maps and $\varepsilon^{-2}$ for 32 regular maps and it is related to the interplay of the noise 33 with the exponential or linear divergence of nearby or34 bits. The present paper provides, on rigorous grounds, 35 the framework suitable to perform a systematic analysis 36 of the statistics of the global error due to roundoff. In a 37 future work and by using the numerical tools developed 38 here, we will compare the results of this paper with those 39 obtained using pure roundoff noise to check if the same 40 qualitative behavior (i.e., the same time scales for the 41 convergence to the asymptotic error distribution) holds. 42

\section{I. INTRODUCTION}

44 The reliability of numerical computations for discrete 45 dynamical systems, where the only source of errors is due to 46 the representation of real numbers by finite strings and to 47 roundoff in arithmetic operations, is a long standing ques48 tion. The algorithms used to implement roundoff arithmetic 49 are not universal but depend on the hardware. As a conse-

\footnotetext{
${ }^{a)}$ Electronic mail: pmarie@cpt.univ-mrs.fr.

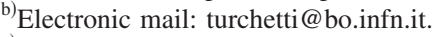

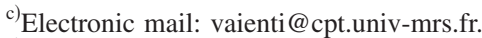

d)Electronic mail: zanlungo@bo.infn.it.
}

quence a rigorous analysis of the propagation of errors due to 50 roundoff in floating point computations is largely 51 incomplete. ${ }^{1}$ The relative error $\eta$ defined by $x^{*}=x(1+\eta), 52$ where $x^{*}$ is the floating point representation of the real num- 53 ber $x$, grows when an iterative procedure is applied and the 54 numerical orbit $x_{n}^{*}=T_{*} x_{n-1}^{*}$ may diverge with respect to the 55 true orbit $x_{n}=T x_{n-1}$, where $T$ is the map acting on the space 56 $X$ and $T_{*}$ is its floating point representation. We also stress 57 that the numerical inverse of a map $T_{*}^{-1}$, computed by imple- 58 menting the algorithm which defines the true inverse $T^{-1}$, is 59 not exact. The distance $d(\cdot, \cdot)$ between the true orbit and the 60 numerical one is in general not known; however if in a single 61 iteration it remains bounded $d\left(x_{n+1}^{*}, T x_{n}^{*}\right) \equiv d\left(T_{*} x_{n}^{*}, T x_{n}^{*}\right)<\varepsilon, 62$ then we say that the numerical orbit is an $\varepsilon$ pseudo-orbit, and 63 some theoretical results are available if the map is mixing. 64

The reversibility error after $n$ iterations $d\left(x_{*}, T_{*}^{-n} \circ T_{*}^{n} x_{*}\right) 65$ has a growth rate comparable to $d\left(T^{n} x_{*}, T_{*}^{n} x_{*}\right)$. Even though 66 arithmetic with roundoff is deterministic it is often assumed 67 that the sequence of errors is random. If so, letting $T x_{n}^{*} 68$ $-T_{*} x_{n}^{*}=\varepsilon \xi_{n}$, the pseudo-orbit $x_{n}^{*}$ appears to be generated by a 69 map $T_{*} x=T x+\varepsilon \xi$ randomly perturbed with an additive noise. 70 To prove that for any given initial point $x_{*}$ the sequence $\xi_{n}$ is 71 actually equivalent to the realization of a random process and 72 to specify its properties is once more a hard theoretical task. 73 Nevertheless it is possible to investigate numerically the sta- $\mathbf{7 4}$ tistics of the pseudorandom sequence $\xi_{n}$ by evaluating $T$ with 75 a much higher accuracy with respect to $T_{*}$ so that we may 76 neglect the difference with the exact result, at least below a 77 significant time scale. To carry out this program it is critical 78 to have some analytical estimates to guide our numerical 79 investigations. In particular we need to know how the statis- 80 tics of global errors $\Delta_{n}=T_{*}^{n} x_{*}-T^{n} x$ changes, evolving from 81 the initial distribution $\rho_{0}(x)=\delta(x)$ to the asymptotic distribu- 82 tion $\rho_{\infty}(x)$. This program differs radically from the search of 83 
84 a true orbit, close to the pseudo-orbit, whose existence is 85 assured by the shadowing lemma. ${ }^{2-8}$

86 In the present work we compare the true orbit $T^{n} x_{0}$ and 87 an $\varepsilon$-pseudo-orbit $\left\{x_{n}^{\prime}\right\}_{n \geq 0}$ starting from the same initial point $88 x_{0}$ and analyze the probability distribution function of the 89 algebraic error $f\left(T^{n} x_{0}\right)-f\left(x_{n}^{\prime}\right)$, where $f$ is a smooth observ90 able over $X$. More specifically we consider the case in which 91 the pseudo-orbit is a random perturbation of the true orbit 92 and provide analytical results to validate the numerical com93 putations. The statistical analysis of actual roundoff errors is 94 postponed to a forthcoming paper.

95 We consider a class $\mathcal{M}$ of dynamical systems with 96 strong mixing properties (see Sec. II for a rigorous defini97 tion) for which exact results are proved and integrable dy98 namical systems as well. The error induced by a random 99 perturbation of the map $T$ in the computation of a smooth 100 observable $f$ is defined by

$101 \Delta_{n}^{\varepsilon}=f\left(T^{n} x\right)-f\left(T_{\omega}^{n} x\right)$,

102 where $T_{\omega}^{n}=T_{\omega_{1}} \circ \cdots \circ T_{\omega_{n}},\left|\omega_{i}\right| \leq \varepsilon$. This random variable is 103 taken with respect to the probability measure given by the 104 direct product of the Lebesgue measure $m$ over $X$ and the 105 measure $\theta_{\varepsilon}^{\mathrm{N}}$ (see Sec. II) over the space of the realizations $\boldsymbol{\omega}$. 106 The characteristic function $\varphi_{n}^{\varepsilon}$ of the random variable $\Delta_{n}^{\varepsilon}$ is 107 simply related to the random classical fidelity, introduced in 108 Ref. 9, which is suited to investigate the statistics of the 109 asymptotic error since it converges pointwise to

$110 \quad \varphi_{n}^{\varepsilon}(u) \rightarrow \mathbb{E}_{\mu}\left(e^{i f u}\right) \overline{\mathbb{E}_{\mu_{\varepsilon}}\left(e^{i f u}\right)}$,

111 where the expectation values are calculated with respect to 112 the invariant measure $\mu$ of the map $T$ and to the stationary 113 measure $\mu_{\varepsilon}$ of the stochastic perturbation $T_{\varepsilon}$ of the map $T$ 114 (the bar denotes complex conjugation, see details in Sec. II). 115 We show that this convergence is exponential with a rate 116 which is related to the rate of decay of correlations. The 117 left-hand side of Eq. (2) is the characteristic function of a 118 random variable which we interpret as the asymptotic error 119 and denote with $\Delta_{\infty}^{\varepsilon}$. This is the main quantity investigated in 120 this paper.

121 The asymptotic error can be computed not only for cha122 otic maps but also for regular maps such as rotations. For the 123 Bernoulli maps of the torus $q x \bmod 1$ and for rotations, a 124 complete analytical study, providing detailed information 125 about the transient to the asymptotic distribution, is possible.

126 Since the asymptotic error is the same for these proto127 types of regular and chaotic maps, the difference must be 128 searched for the transient, which reflects the divergence rate 129 of nearby unperturbed orbits. In particular the asymptotic 130 error is reached superexponentially fast for the Bernoulli 131 map and just exponentially fast for rotations; in the first case 132 the transition is very sharp, whereas it is smooth in the sec133 ond case. The time scales for the convergence to the 134 asymptotic error distribution are $n \sim \log (1 / \varepsilon)$ and $n=\varepsilon^{-2}$, re135 spectively. We also investigate, mostly numerically, systems 136 with "intermediate" ergodic properties, such as intermittent 137 maps, the logistic map, the Hénon attractor, and the standard 138 map.

139 The plan of the paper is the following. Section II is 140 devoted to analytical results for the class $\mathcal{M}$. In Sec. III we present the analytical results on two prototypes of regular 141 and chaotic maps and compare them with numerical compu- 142 tations. In Sec. IV we present the results of numerical com- 143 putations for several systems, where analytical results are 144 missing: in particular we consider fractal attractors and fol- 145 low the evolution looking at observables with a Cantorian 146 structure. For these systems also the density distribution 147 function for the asymptotic error has a fractal shape. Our 148 major achievements are summarized in Sec. V and technical 149 details are explained in Appendices A-D.

\section{ERROR STATISTICS}

\section{A. Random perturbations and fidelity}

Let $X$ be a compact Riemannian manifold equipped with 153 the Lebesgue measure $m$ and consider a Borel measurable 154 map $T: X \rightarrow X$ admitting a physical measure $\mu$ (very often 155 also called SRB measure from Sinai, Ruelle, and Bowen who 156 introduced it), which is defined through the limit

$$
\lim _{n \rightarrow \infty} \int \psi \circ T^{n} d m=\int \psi d \mu,
$$

where $\psi$ is any continuous function over $X$. We introduce the 159 random perturbation of the preceding system and succes- 160 sively we consider the fidelity as an interesting quantity to 161 characterize the annealed correlation integrals arising under 162 the stochastic perturbation.

163

Let $\left(\omega_{k}\right)_{k \in \mathbb{N}}$ be a sequence of independent and identically 164 distributed (iid) random variables with values in the vector 165 space $\Omega_{\varepsilon}$ and with distribution $\theta_{\varepsilon}$. To each $\omega \in \Omega_{\varepsilon}$ we asso- 166 ciate a map $T_{\omega}$ with $T_{0}=T$ and define the iterated perturbed 167 map as $T_{\omega_{n}} \circ \cdots \circ T_{\omega_{1}} \equiv T_{\omega}^{n}$.

According to Ref. 9 we consider a class of maps $\mathcal{M}$ such 169 that the following are taken into account.

170

The iterated perturbed map admits an invariant sta- 171 tionary measure $\mu_{\varepsilon}$ defined by

172

$\lim _{n \rightarrow \infty} \int_{X} \int_{\Omega_{\varepsilon}^{\mathrm{N}}} \psi\left(T_{\boldsymbol{\omega}}^{n} x\right) d \theta_{\varepsilon}^{\mathrm{N}}(\boldsymbol{\omega}) d m(x)=\int \psi d \mu_{\varepsilon}$.

The distribution $\theta_{\varepsilon}$ is chosen in such a way that $\mu_{\epsilon} 174$ will be absolutely continuous with respect to $m$ (see 175 Sec. II B), and moreover $\int_{X} \psi d \mu_{\varepsilon} \rightarrow \int_{X} \psi d \mu$ when 176 $\varepsilon \rightarrow 0$ (stochastic stability).

(ii) The correlation integral decays exponentially on the 178 space of $\mathcal{C}^{1}$ observables, i.e.,

$$
\begin{aligned}
& \left|\int_{X} \psi_{1}\left(T^{n} x\right) \psi_{2}(x) d m(x)-\int_{X} \psi_{1} d \mu \int_{X} \psi_{2} d m\right| \\
& \quad \leq C \lambda^{-n}\left\|\psi_{1}\right\|_{1}\left\|\psi_{2}\right\|_{2},
\end{aligned}
$$

where $C>0$ and $\lambda>1$ are determined only by the 182 map $T$ and the two norms depend on the map and on 183 the space of observables (Ref. 9).

(iii) An exponential decay for the annealed correlation in- 185 tegral is assumed still for $\mathcal{C}^{1}$ functions, 


$$
\begin{aligned}
& \mid \int_{X} \int_{\Omega_{\varepsilon}^{\mathrm{N}}} \psi_{1}\left(T_{\boldsymbol{\omega}}^{n} x\right) \psi_{2}(x) d \theta_{\varepsilon}^{\mathrm{V}}(\boldsymbol{\omega}) d m(x) \\
& \quad-\int_{X} \psi_{1} d \mu_{\varepsilon} \int_{X} \psi_{2} d m \mid \\
& \leq C \lambda^{-n}\left\|\psi_{1}\right\|\left\|_{1}\right\| \psi_{2} \|_{2},
\end{aligned}
$$

190 where $C, \lambda$, and the norms 1 and 2 are the same as in 191 Eq. (5).

192 We introduce the classical fidelity by means of the fol193 lowing integral (this definition was inspired by Ref. 10):

194

$$
F_{n}^{\varepsilon}=\int_{X} \int_{\Omega_{\varepsilon}^{\mathrm{N}}} \psi_{1}\left(T_{\boldsymbol{\omega}^{n}}^{n}\right) \psi_{2}\left(T^{n} x\right) d \theta_{\varepsilon}^{\mathrm{N}}(\boldsymbol{\omega}) d m(x),
$$

195 where $\psi_{1}$ and $\psi_{2}$ are $C^{1}$ functions.

196 It is possible to prove the following theorem (Ref. 9).

197 Theorem 1: For the class $\mathcal{M}$ there exists a constant $198 C_{1}>0$ for which

$199 \quad\left|F_{n}^{\varepsilon}-\int_{X} \psi_{1} d \mu_{\varepsilon} \int_{X} \psi_{2} d \mu\right|$

200

$$
\leq C_{1} \varepsilon^{-\kappa} \lambda^{-n}\left(\left\|\psi_{2}\right\|_{1}\left\|\psi_{1}\right\|_{C^{0}}+\left\|\psi_{1}\right\|_{1}\left\|\psi_{2}\right\|_{C^{0}}\right)
$$

201 where $\|\cdot\|_{1}$ is a suitable norm on the space of observables 202 and $\|\cdot\|_{C^{0}}$ is the supremum norm on continuous functions.

\section{B. Additive noise}

204 In this article we restrict ourselves to a particular random 205 perturbation, the additive noise, which satisfies the assump206 tions of Theorem 1 and is well defined whenever the space $X$ 207 is the $d$-dimensional torus $X=\mathrm{T}^{d}$. In this case we define the 208 random maps as $T_{\omega}=T x+\omega \bmod \mathbb{T}^{d}$, where $\omega \in \mathbb{T}^{d}$, and then 209 take $\theta_{\varepsilon}$ absolutely continuous with respect to the Lebesgue 210 measure $d \omega$ over $T^{d}$ and with a bounded density $h_{\varepsilon}$ with 211 support contained in the square $[-\varepsilon, \varepsilon]^{d}$ and such that $\int d \theta_{\varepsilon}$ $212=\int h_{\varepsilon}(\omega) d \omega=1$. Letting $H(\xi)$ be a non-negative bounded 213 function with support on $[-1,1]^{d} \subset \mathbb{R}^{d}$ such that $\int H(\xi) d \xi$ $214=1$, the function $h_{\varepsilon}(x)$ is defined by $h_{\varepsilon}(\omega)=\varepsilon^{-d} H(\omega / \varepsilon)$. The 215 change in variable $\omega=\varepsilon \xi$ shows that the density $h_{\varepsilon}(\omega)$ of the 216 measure $\theta_{\varepsilon}(\omega)$ has the required properties. For the maps of 217 the torus $\mathbb{T}$ discussed in Sec. III we consider a sequence of 218 iid random variables $\left(\xi_{k}\right)_{k \in \mathbb{N}}$ in $[-1,1] \subset \mathbb{R}$ and a function $219 H(\xi)$ equal to $1 / 2$ for $|\xi| \leq 1$ and equal to 0 for $|\xi|>1$, limit of 220 a sequence of normalized functions with support in $\xi \in[$ $221-1,1]$. As a consequence after the change $\omega=\varepsilon \xi$ the fidelity 222 we compute reads

$$
F_{n}^{\varepsilon}=2^{-d} \int_{0}^{1} d x \int_{-1}^{1} d \xi \psi_{1}\left(T^{n} x\right) \psi_{2}\left(T_{\varepsilon \xi_{n}} \circ \cdots \circ T_{\varepsilon \xi_{1}} x\right) .
$$

224 When the phase space $X$ is a subset $D \in \mathbb{R}^{n}$, we require that 225 the image of $D$ is strictly included in $D$ and that it remains so 226 when randomly perturbed. It is straightforward to check that 227 the orbits generated by maps perturbed with additive noise 228 are $\varepsilon$ pseudo-orbits. The equivalence between pseudo-orbits 229 and random orbits holds for a wider class of noises, see 230 Ref. 11.
Remark 1: Reference 9 gives several examples of $d y$ - 231 namical systems satisfying Theorem 1: Anosov diffeomor- 232 phisms, uniformly hyperbolic attractors, piecewise expand- 233 ing maps of the interval, and uniformly hyperbolic maps with 234 singularities. Theorem 1 could be generalized by considering 235 two randomly iterated perturbed maps $T_{\boldsymbol{\omega}}^{n}$ and $T_{\boldsymbol{\omega}^{\prime}}^{n}$ having 236 stationary measures $\mu_{\varepsilon}$ and $\mu_{\varepsilon}^{\prime}$. Equation (8), where we re- 237 place $T^{n}$ with $T_{\boldsymbol{\omega}^{\prime}}^{n}$ and $\mu$ with $\mu_{\varepsilon}^{\prime}$, would still hold. This result 238 allows us to compare a randomly perturbed map with an- 239 other one having a smaller perturbation when the exact map 240 is not available as it happens in numerical computations.

241

\section{Error distribution}

We consider the dynamical system $(X, T, \mu)$, where $\mu$ is 243 a physical measure, and the family of random transforma- 244 tions $T_{\omega_{k}}$ previously defined. Let $f: X \rightarrow \mathbb{R}$ be a $\mathcal{C}^{1}$ observable 245 and $m \otimes \theta_{\varepsilon}^{N}$ be the probability measure on the product space 246 $X \times \Omega_{\varepsilon}^{N}$. The basic quantity investigated in this paper is the 247 algebraic error

248

$$
\Delta_{n}^{\varepsilon}=f\left(T^{n} x\right)-f\left(T_{\omega}^{n} x\right)
$$

given by the difference of two stochastic processes, for 250 which we now state the following result that we use to in- 251 vestigate asymptotic distributions of errors.

252

Theorem 2: Suppose the map $T \in \mathcal{M}$; then when $n$ goes 253 to $+\infty$, the random process $\Delta_{n}^{\varepsilon}$, whose characteristic func- 254 tion is $\varphi_{n}^{\varepsilon}(u)$, converges in distribution to a random variable 255 $\Delta_{\infty}^{\varepsilon}$ whose characteristic function is given by

256

$$
\varphi_{\infty}^{\varepsilon}(u)=\lim _{n \rightarrow \infty} \varphi_{n}^{\varepsilon}(u)=\int_{X} e^{i u f} d \mu \int_{X} e^{-i u f} d \mu_{\varepsilon} .
$$

Proof: Indeed writing the characteristic function of the 258 variable $\Delta_{n}^{\varepsilon}$ as $\varphi_{n}^{\varepsilon}(u)$ and using Fubini theorem, we have

$$
\begin{aligned}
\varphi_{n}^{\varepsilon}(u) & \equiv \int_{X \times \Omega_{\varepsilon}^{\mathrm{N}}} e^{i u \Delta_{n}^{\varepsilon}(x, \boldsymbol{\omega})} d m(x) d \theta_{\varepsilon}^{\mathrm{N}}(\boldsymbol{\omega}) \\
& =\int_{X} \int_{\Omega_{\varepsilon}^{\mathrm{N}}} e^{i u\left[f\left(T^{n} x\right)-f\left(T_{\boldsymbol{\omega}}^{n} x\right)\right]} d \theta_{\varepsilon}^{\mathrm{N}}(\boldsymbol{\omega}) d m(x) .
\end{aligned}
$$

By Theorem 1 and by choosing $\psi_{1}(x)=e^{i u f(x)}, \psi_{2}(x)=e^{-i u f(x)} 262$ we immediately get the equality (10). Let us now denote 263 with $\Phi_{n}^{\varepsilon}$ the distribution function of the variable $\Delta_{n}^{\varepsilon}$; if we 264 can prove that $\varphi_{\infty}^{\varepsilon}$ is continuous at $u=0$, then by Theorem 3, 265 p. 266 in Ref. 12, we can conclude that $\varphi_{\infty}^{\varepsilon}$ is the character- 266 istic function of some distribution function $\Phi_{\infty}^{\varepsilon}$ and $\Phi_{n}^{\varepsilon}$ con- 267 verges completely to $\Phi_{\infty}^{\varepsilon}$. In this case we will say that the 268 random variables $\Delta_{n}^{\varepsilon}$ converge in distribution or law to a 269 random variable, which we denote with $\Delta_{\infty}^{\varepsilon}$ and which has 270 $\Phi_{\infty}^{\varepsilon}$ as distribution function.

271

Since $\left|e^{ \pm i u f}\right| \leq 1$ and $\mu$ and $\mu_{\varepsilon}$ are probability measures, 272 by the dominated convergence theorem we immediately have 273 that $\lim _{u \rightarrow 0} \varphi_{\infty}^{\varepsilon}(u)=1=\varphi_{\infty}^{\varepsilon}(0)$, which proves that $\varphi_{\infty}^{\varepsilon}$ is con- 274 tinuous at 0 .

We are not really interested in the random variable $\Delta_{\infty}^{\varepsilon}, 276$ but rather in its distribution function $\Phi_{\infty}^{\varepsilon}$. Whenever 277 $\int_{-\infty}^{\infty}\left|\varphi_{\infty}^{\varepsilon}(u)\right| d u<\infty$, the Lévy inversion formula asserts that the 278 
279 distribution function $\Phi_{\infty}^{\varepsilon}$ is absolutely continuous with a 280 bounded continuous density $\rho_{\infty}^{\varepsilon}$ [probability density function 281 (pdf)] given by

282

$$
\rho_{\infty}^{\varepsilon}(t)=\frac{1}{2 \pi} \int_{-\infty}^{\infty} e^{-i u t} \varphi_{\infty}^{\varepsilon}(u) d u .
$$

283 We also note that the convergence to the asymptotic charac284 teristic function is exponential but not uniform in the noise

285

$$
\left|\varphi_{n}^{\varepsilon}(u)-\varphi_{\infty}^{\varepsilon}(u)\right|=\mathcal{O}\left(\varepsilon^{-\kappa} \lambda^{-n}\right) .
$$

286 This point is further investigated in Secs. III and IV and in 287 Appendix D.

288 Remark 2: Since our random variable $\Delta_{n}^{\varepsilon}$ is bounded on $289 X$, all its moments are uniformly integrable and the moments 290 of $\Delta_{\infty}^{\varepsilon}$ are given by the limit, for $n \rightarrow \infty$, of the corresponding 291 moments of $\Delta_{n}^{\varepsilon}$. In particular, we have

(i) $\mathbb{E}_{m \otimes \theta_{\varepsilon}}\left(\Delta_{\infty}^{\varepsilon}\right)=\mathbb{E}_{\mu}(f)-\mathbb{E}_{\mu_{\varepsilon}}(f)$

293

ii)

$$
\begin{aligned}
\mathrm{V}_{m \otimes \theta_{\varepsilon}^{\mathrm{N}}}\left(\Delta_{\infty}^{\varepsilon}\right) & =\mathrm{V}_{\mu}(f)+\mathrm{V}_{\mu_{\varepsilon}}(f) \\
= & \int f^{2} d \mu-\left(\int f d \mu\right)^{2} \\
& +\int f^{2} d \mu_{\varepsilon}-\left(\int f d \mu_{\varepsilon}\right)^{2},
\end{aligned}
$$

296 where $\mathbb{E}_{\nu}$ (respectively $V_{\nu}$ ) denotes the expectation (respec297 tively the variance) with respect to the measure $\nu$. Since we 298 have assumed $\mu_{\varepsilon} \rightarrow \mu$ (stochastic stability), the average of 299 the asymptotic error vanishes when $\varepsilon \rightarrow 0$, whereas its vari300 ance remains finite. This is a memory phenomenon because 301 even if the noise vanishes after the system has evolved its 302 effect persists. This is another consequence of the stochastic 303 instability of the classical fidelity pointed out in Ref. 9. Ad304 ditional considerations may be found in Appendix A, where 305 we compare our results with a recent related work by Sauer.

\section{III. ANALYTICAL RESULTS ON PROTOTYPE 307 MAPS}

\section{A. Chaotic maps and rotations on the circle}

309 As an easy illustration of the theorem 2 we consider the 310 map $T$ defined on one-dimensional (1D) torus $X=\mathbb{T}^{1}$ by $T x$ $311=q x \bmod 1$, where $q \geq 2$ is a positive integer. We perturb it 312 with additive noise producing the random transformations $313 T_{\varepsilon \xi}(x)=q x+\varepsilon \xi \bmod 1$, where $\varepsilon>0$ is the noise intensity and $314 \xi$ is a random variable uniformly distributed over $[-1,1]$. By 315 inspection of formula (4), it is easy to check that the noise 316 just introduced has a stationary measure which coincides 317 with the Lebesgue measure on $\mathrm{T}^{1}$. The map $T$ clearly belongs 318 to $\mathcal{M}$ and theorem 2 holds for smooth observables. We shall 319 rather consider the observable identity $f(x)=x$ which is dis320 continuous on the torus. A direct proof of convergence will 321 be given by using Fourier series, and we show in Sec. III B 322 that the characteristic function of the asymptotic error is $323 \varphi_{\infty}^{\varepsilon}(u)=2 u^{-2}(1-\cos u)$ [in agreement with Eq. (2)]. The 324 asymptotic error is the triangular function on $[-1,1]$, namely, $325 \rho_{\infty}^{\varepsilon}(t)=(1-|t|) 1_{[-1,1]}$.
We apply the same Fourier analysis to regular maps, 326 such as the translations on the torus $T x=x+\chi \bmod 1$, which 327 correspond to rotations of angle $2 \pi \chi$ on the circle $\mathcal{C}^{1}$, and we 328 perturb it with additive noise [we stress that for such maps 329 our theorem 2 does not apply]. The random map iterated $n 330$ times reads

$$
T_{\varepsilon \xi}^{n} \equiv x+n \chi+\varepsilon\left(\xi_{1}+\xi_{2}+\cdots+\xi_{n}\right) \bmod 1,
$$

where the $\left(\xi_{l}\right)_{l \geq 1}$ are iid random variables with common uni- 333 form distribution over $[-1,1]$. The Lebesgue measure on ${ }^{1}{ }^{1} 334$ is the unique stationary measure for the process. Let us con- 335 sider the fidelity [Eq. (9)] $F_{n}^{\varepsilon}$ for the function $\psi=\psi_{1}=\psi_{2}^{*}$ with 336 Fourier coefficients $\left(\psi_{k}\right)_{k \in Z}$. The function $\psi$ is taken piece- 337 wise $C^{1}$ (at least) with a finite number of discontinuity 338 points. Simple calculations give (see Appendix B)

339

$$
F_{n}^{\varepsilon}=\left|\psi_{0}\right|^{2}+\sum_{k \neq 0}\left|\psi_{k}\right|^{2} S^{n}(k \varepsilon), \quad S(x)=\frac{\sin (2 \pi x)}{2 \pi x} .
$$

The function $S$ can be bounded by

$$
|S(x)| \leq \begin{cases}e^{-\ln (2 \pi) x^{2}} & \text { if }|x|<1 \\ \frac{1}{2 \pi|x|} & \text { if }|x| \geq 1 .\end{cases}
$$

Using this bound in Eq. (14) and since the Fourier coeffi- 343 cients are at least bounded by $\left|\psi_{k}\right| \leq C|k|^{-1}$, where $C$ is a 344 constant depending on the observable $\psi$, we get, after a few 345 manipulations (see Appendix B for details),

346

$$
\left.\left|F_{n}^{\varepsilon}-\right| \psi_{0}\right|^{2} \mid \leq C^{2}\left((2-\varepsilon) e^{-\varepsilon^{2} n(\ln (2 \pi))}+2 \varepsilon(2 \pi)^{-n}\right) .
$$

This result applies also to $\varphi_{n}^{\varepsilon}(u)$, which is the fidelity com- 348 puted using $\psi=e^{i u f(x)}$.

\section{B. Transients for the maps on the torus}

350

Since Eq. (16) converges to zero and by taking $f(x)=x, 351$ the asymptotic distribution of errors $\Delta_{\infty}^{\varepsilon}$ for rotations is again 352 the triangular distribution, as in the case of $q x \bmod 1$ with 353 $q \geq 2$. Therefore a chaotic and a very regular map (which is 354 the identity for $\chi=0$ ) share the same distribution for the error 355 between the true orbit and its random perturbation with the 356 same initial condition. This is not surprising if one realizes 357 that what we are doing for the observable identity $f(x)=x$ is 358 just to compute the distribution of the difference of two 359 points selected randomly, and independently one from the $\mathbf{3 6 0}$ other, on the circle with uniform distribution: the distribution 361 of the difference is just the triangular law. The previous re- 362 quired statistical assumptions are guaranteed by the Ber- 363 noulli property of the map $q x \bmod 1$ and by the indepen- 364 dence and the common uniform distribution of the kicks $\xi_{l} 365$ for rotations.

366

The very different ergodic properties of the two systems 367 are reflected, however, in the rate of convergence to the lim- 368 iting distribution. Equation (12) suggests an exponential de- 369 cay for $\mathcal{M}$ maps [for $q x \bmod 1$ the exponent is $\kappa=1$ (Ref. 370 $9)$ ], but we can considerably improve the latter estimate us- 371 ing Fourier series and prove that the convergence for 372 $q x$ mod 1 is indeed superexponential. We first observe that 373 


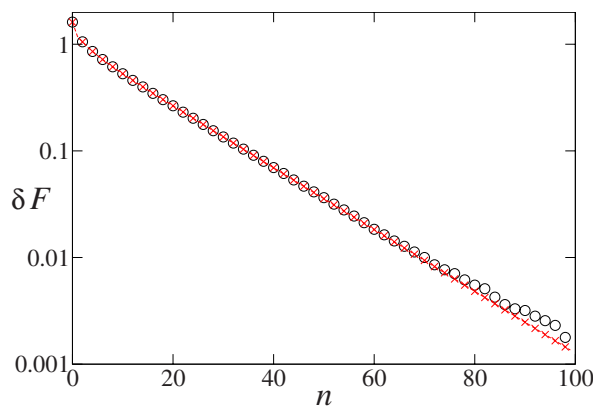

FIG. 1. (Color online) Comparison between the analytical result, Eq. (14), and Monte Carlo integrals for the decay of fidelity [Eq. (24)] in rotations, $\varepsilon=0.1$ (analytical result: red dashed line and crosses; Monte Carlo: circles). We used $N=10^{7}$ integration points in the Monte Carlo method. The test functions $\psi_{1}=\psi_{2}^{*}$ were defined in Fourier space as $\psi_{k}=k^{-1}$ truncated at $k$ $=30$.

$$
F_{n}^{\varepsilon}=\left|\psi_{0}\right|^{2}+\sum_{k \neq 0}\left|\psi_{k}\right|^{2} S_{n, q}(k \varepsilon)
$$

375 where we have defined

$$
376 \quad S_{n, q}(x)=\prod_{k=0}^{k=n-1} S\left(q^{k} x\right)
$$

377 (the proof of this equation is given in Appendix B). A bound 378 for Eq. (17) can be found as

379

$$
\left.\left|F_{n}^{\varepsilon}-\right| \psi_{0}\right|^{2} \mid \leq \begin{cases}A e^{-\alpha q^{2\left(n-n_{*}\right)}} & \text { if } n<n_{*} \\ A e^{-\alpha} q^{-\left(n-n_{*}\right)^{2} / 2} & \text { if } n \geq n_{*},\end{cases}
$$

380 where

$$
381 \varepsilon q^{n_{*}}=1 \quad \text { or } n_{*}=-\log _{q} \varepsilon .
$$

382 This formula shows a sharp transition in the decay whenever $383 n \sim \ln \varepsilon^{-1} / \ln q=n_{*}$ and the decay becomes superexponential. 384 Instead, for rotations, such a transition point does not exist 385 and the decay is smooth, see Figs. 1 and 2. The difference 386 between these two regimes becomes also apparent when we 387 compute $\rho_{n}^{\varepsilon}$, the distribution function of $\Delta_{n}^{\varepsilon}$, for the identity 388 observable, which is given by the inverse Fourier transform 389 of the following characteristic functions that we obtain after 390 evaluating the Fourier coefficients

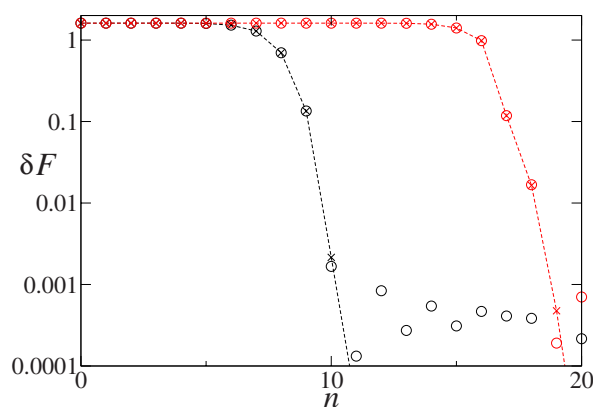

FIG. 2. (Color online) Comparison between the analytical result, Eq. (17), and Monte Carlo integral for the decay of fidelity [Eq. (24)] in $3 x \bmod 1$. $\varepsilon=10^{-4}$ in black and $\varepsilon=10^{-8}$ in red (gray in black and white) (analytical result: dashed line and crosses; Monte Carlo: circles). We used $N=10^{7}$ integration points in the Monte Carlo method. The test functions $\psi_{1}=\psi_{2}^{*}$ were defined in Fourier space as $\psi_{k}=k^{-1}$ truncated at $n=30$.

$$
\psi_{k}=e^{i(u / 2-\pi k)} \frac{\sin (u / 2-\pi k)}{u / 2-\pi k}
$$

of the function $\psi(x)$ defined on the torus so that $\psi(x)=e^{i u x}$ for 392 $0 \leq x<1$, namely,

$$
\psi(x)= \begin{cases}e^{i u(x-[x])} & \text { if } x>0 \\ e^{i u(1+x-[x])} & \text { if } x<0,\end{cases}
$$

where $[x]$ stands for the integer part of $x \in \mathbb{R}$ (this choice is 395 equivalent to study $e^{i u x}$ defined on the torus). The result ex- 396 plicitly reads

$\varphi_{n}^{\varepsilon}(u)=\frac{2}{u^{2}}(1-\cos u)$

$$
+\sum_{k=1}^{\infty} S^{n}(k)\left[\frac{\sin ^{2}(u / 2-\pi k)}{(u / 2-\pi k)^{2}}+\frac{\sin ^{2}(u / 2+\pi k)}{(u / 2+\pi k)^{2}}\right]
$$

for rotations and

$$
\begin{aligned}
\varphi_{n}^{\varepsilon}(u)= & \frac{2}{u^{2}}(1-\cos u) \\
& +\sum_{k=1}^{\infty} S_{n, q}(k)\left[\frac{\sin ^{2}(u / 2-\pi k)}{(u / 2-\pi k)^{2}}+\frac{\sin ^{2}(u / 2+\pi k)}{(u / 2+\pi k)^{2}}\right]
\end{aligned}
$$

for $q x \bmod 1$. See Appendix B for more details. The inverse 403 Fourier transform of $2 u^{-2}(1-\cos u)$ is the triangular distribu- 404 tion so that

$$
\rho_{\infty}^{\varepsilon}(t)=(1-|t|) \rrbracket_{[-1,1]} .
$$

The continuum limit of these processes is described in 407 Appendix C.

\section{Comparison to numerical results}

We have compared the analytical results with the output 410 of numerical computations based essentially on Eq. (9). The 411 decay of fidelity [see also Eq. (8)] on $X=[0,1]$ is defined by 412

$$
\begin{aligned}
\delta F_{n}^{\varepsilon}= & F_{n}^{\varepsilon}-2^{-d}\left(\int_{0}^{1} d x \psi_{1}\left(T^{n}(x)\right)\right) \\
& \times\left(\int_{0}^{1} d x \int_{-1}^{1} d \xi \psi_{2}\left(T_{\varepsilon \xi_{n}} \circ \cdots \circ T_{\varepsilon \xi_{1}} x\right)\right),
\end{aligned}
$$

where $F_{n}^{\varepsilon}$ is given by Eq. (9). The integrals are performed 415 randomly choosing $N$ vectors $(x, \xi)$ in $\mathbb{R}^{d(n+1)}$, i.e., using a 416 Monte Carlo method whose accuracy is of order $N^{-1 / 2}$. For 417 smooth test functions, integration methods with regularly 418 distributed $x$ points can improve the accuracy, but since for $\mathbf{4 1 9}$ chaotic maps the smoothness of the integrand is rapidly lost $\mathbf{4 2 0}$ and integration error estimates are difficult we systematically 421 use a Monte Carlo on $(x, \boldsymbol{\xi})$ for which the error estimate 422 $N^{-1 / 2}$ always holds. We show the comparison between the 423 analytical and numerical results on the decay of fidelity for $\mathbf{4 2 4}$ rotations (Fig. 1) and for the map $3 x$ mod 1 (Fig. 2); while in 425 Figs. 3-6 we compare the analytical results for $\rho_{n}^{\varepsilon}$ [Fourier 426 inversion of Eqs. (22) and (23)] with the corresponding nu- 427 merical computations (i.e., with a Monte Carlo sampling of $\mathbf{4 2 8}$ the error $\Delta_{n}^{\varepsilon}$ in which the probability function $\rho_{n}^{\varepsilon}$ is approxi- 429 

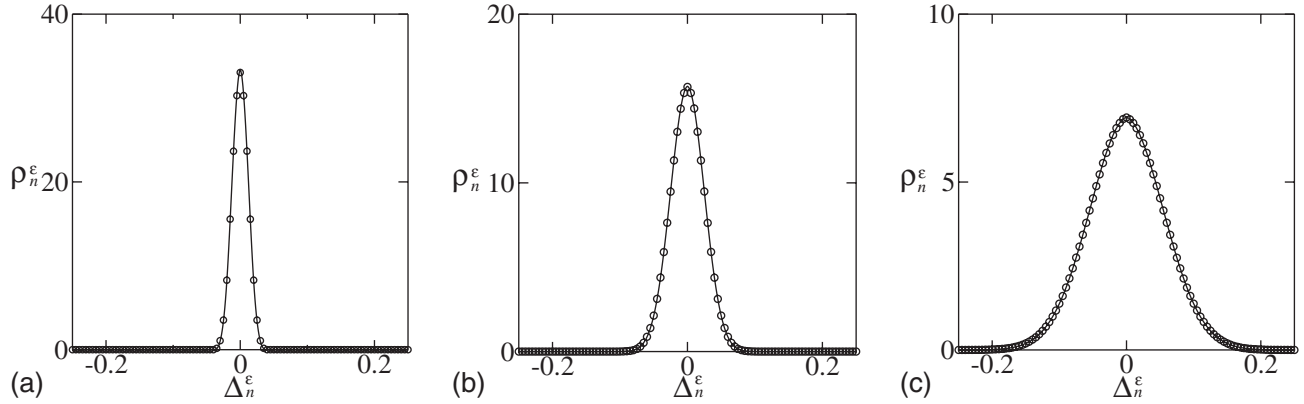

FIG. 3. Comparison between the analytical result for $\rho_{n}^{\varepsilon}$ in rotations, Fourier transform of Eq. (22) (continuous line), and Monte Carlo sampling (circles), $\varepsilon=10^{-2}$. (a) $n=4$; (b) $n=19$; (c) $n=99$.
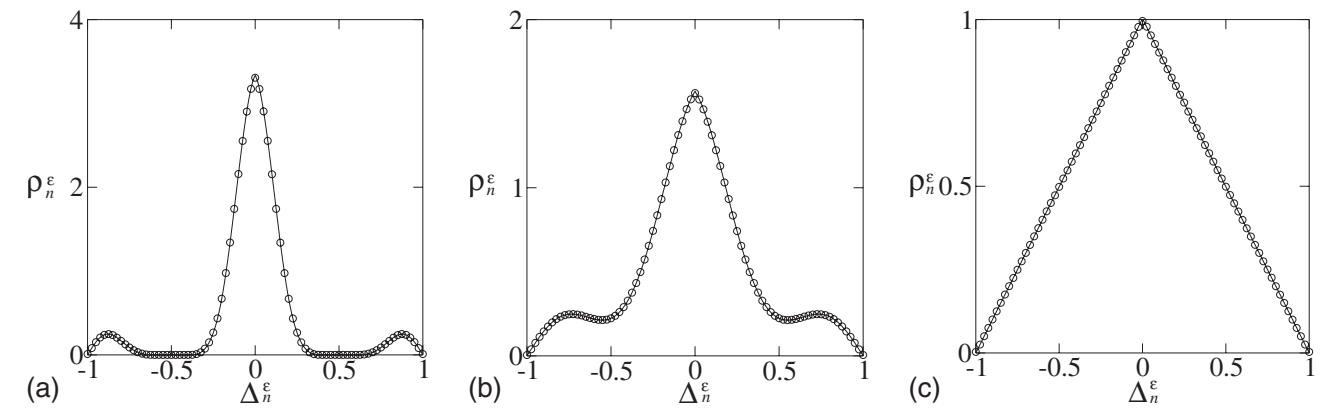

FIG. 4. Comparison between the analytical result for $\rho_{n}^{\varepsilon}$ in rotations, Fourier transform of Eq. (22) (continuous line), and Monte Carlo sampling (circles), $\varepsilon=0.1$. (a) $n=4$; (b) $n=19$; (c) $n=99$. Compare the transition times with Fig. 1 .
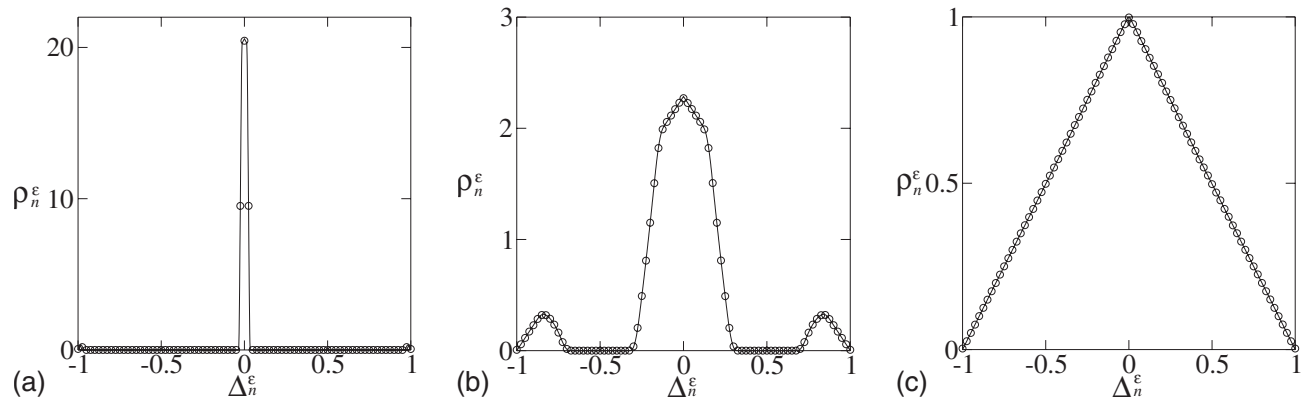

FIG. 5. Comparison between the analytical result for $\rho_{n}^{\varepsilon}$ in $3 x \bmod 1$, Fourier transform of Eq. (23) (continuous line), and Monte Carlo sampling (circles), $\varepsilon=10^{-4}$. (a) $n=6$; (b) $n=8$; (c) $n=10$. Compare the transition times with Fig. 2.
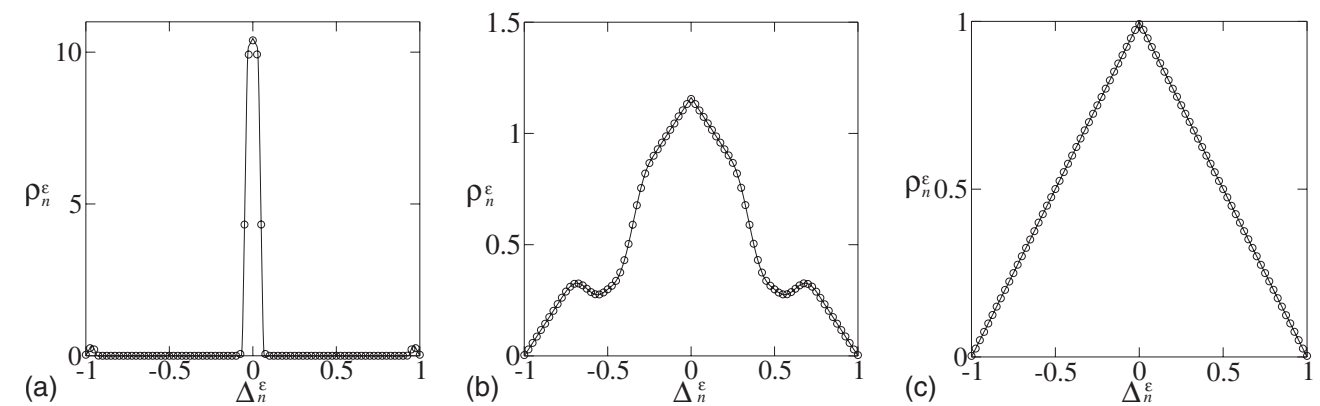

FIG. 6. Comparison between the analytical result for $\rho_{n}^{\varepsilon}$ in $3 x \bmod 1$, Fourier transform of Eq. (23) (continuous line), and Monte Carlo sampling (circles), $\varepsilon=10^{-8}$. (a) $n=15$; (b) $n=17$; (c) $n=19$. Compare the transition times with Fig. 2. 
430 mated by the probability of $\Delta_{n}^{\varepsilon}$ to be in an interval of length $431 \Delta x)$.

432 These figures show clearly that while for rotations the 433 transition from the initial condition $\rho_{0}^{\varepsilon}(x)=\delta(x)$ to the 434 asymptotic (triangular) distribution is a gradual process, 435 whose time scale is of order $\varepsilon^{-2}$, for the map $3 x \bmod 1$ we 436 have a sharp transition that starts around $n_{*}=-\log _{q} \varepsilon$ [see 437 Eq. (19)]. In the transition region the slope does not depend 438 on $\varepsilon$ but is related to the divergence of nearby orbits, namely, 439 to the decay rate of correlations $\lambda$ or the maximum Lyapunov 440 exponent $\Lambda$ (for several systems $\lambda=e^{\Lambda}$ ). According to 441 Eq. (20) when $\varepsilon$ varies from $10^{-4}$ to $10^{-8}$ the transition $n_{*}$ 442 varies by a factor of 2 . For $n \ll n_{*}$ the error distribution func443 tion can be well approximated by a $\delta$ function, i.e., the per444 turbed system can be considered as equivalent to the unper445 turbed one.

446 In both regular and chaotic maps the asymptotic distri447 bution is reached when $\delta F_{n}$ approaches a value a few order 448 of magnitude lower than the initial value. We remark that $449 \rho_{n}^{\varepsilon}(x)$ is the Fourier transform of $\varphi_{n}^{\varepsilon}(u)$, i.e., of the fidelity 450 computed using $\psi_{1}=\psi_{2}^{*}=e^{i u x}$. The comparison between the 451 results in Figs. 1 and 2 and those in Figs. 3-6 suggests (as 452 we have verified analyzing different test functions) that the 453 decay of fidelity depends very weakly on the functions $\psi_{1}$, $454 \psi_{2}$, and thus that the analysis of a single, easily computable 455 test function provides information about the evolution of $456 \rho_{n}^{\varepsilon}(x)$.

\section{IV. NUMERICAL COMPUTATIONS}

$458 \quad$ In this section we apply the theory developed above to 459 various dynamical systems; for the majority of them it is not 460 known whether all the conditions required to belong to the 461 class $\mathcal{M}$ are satisfied. We compute numerically the distribu462 tion of the error $\rho_{n}^{\varepsilon}$ for additive noise. The chosen observ463 ables are $f(x)=x$ for 1D maps and $f(\mathbf{x})=x, f(\mathbf{x})=y$ for two464 dimensional (2D) maps. Whenever $x$ parametrizes a linear 465 subspace $S$ of $\mathbb{R}$, the difference $x_{1}-x_{2}$ is the distance between $466 x_{1}$ and $x_{2}$ up to a sign. The integrals with respect to the 467 physical measure and the stationary measure are performed 468 by using their definition as weak limits of the Lebesgue mea469 sure. These limiting measures are no longer equal to the 470 Lebesgue measure as in the examples in Sec. III. Two rel471 evant classes of maps are considered. In the first one, which 472 includes the maps with neutral fixed point(s) and the logistic 473 map, the measures are absolutely continuous with respect to 474 Lebesgue. In the second class, which includes the Hénon and 475 the Baker's maps, the invariant measures are singular with 476 respect to Lebesgue. For these maps the attractor has a frac477 tal structure, which is locally described as the product of a 478 smooth manifold with a Cantor set. When we deal with maps 479 which are not hyperbolic and admit an absolutely continuous 480 invariant measure with a density which is summable but not 481 necessarily bounded (this is notably the case of the intermit482 tent and quadratic maps), or when we have singular invariant 483 measures as for the Hénon and the Baker's maps, we are not 484 anymore sure that the characteristic function $\varphi_{\infty}^{\varepsilon}$ is Lebesgue integrable. We remind that this is a sufficient condition in 485 order to apply the Lévy inversion formula (11). In Appendix 486 $\mathrm{D}$ we explain what happens when we lose the summability of 487 the characteristic function.

488

The numerical analysis consists of four steps.

489

- Decay of fidelity in a test function. On the basis of the 490 results obtained for translations on the torus and for the 491 map $q x$ mod 1, we study the decay of fidelity [Eq. (24)] for 492 the test function $\psi(x)=x$ ( $x$ observable) using a Monte 493 Carlo method to compute the integrals. This analysis pro- 494 vides the values of $n$ at which the transition from $\rho_{0}^{\varepsilon} 495$ $=\delta(x)$ to the asymptotic regime $\rho_{\infty}^{\varepsilon}$ occurs.

496

- Computation of $\rho_{n}^{\varepsilon}$ for significant values of $n$. Using a 497 Monte Carlo sampling we perform the statistical analysis 498 of $\Delta_{n}^{\varepsilon}$, estimating its distribution $\rho_{n}^{\varepsilon}$, for the values of $n 499$ suggested by the analysis in the first step.

500

- Asymptotic limit. The two previous steps allow us to iden- 501 tify a value $\bar{n}(\varepsilon)$ beyond which $\rho_{n}^{\varepsilon}$ has reached its 502 asymptotic value. We thus compute with high accuracy $\rho_{\infty}^{\varepsilon} 503$ as the average $\overline{\rho_{n^{\prime}}^{\varepsilon}}$ over several values $n^{\prime}>\bar{n}$.

504

- Check of the validity of Eq. (2). We compute $\varphi_{\infty}^{\varepsilon}$ using Eq. 505 (2), and its Fourier transform, to check if it coincides with 506 $\rho_{\infty}^{\varepsilon}$ that is computed with a Monte Carlo sampling, as pre- 507 dicted by Theorem 2 .

508

Remark 3: In numerical computations, we cannot evalu- 509 ate the error $\Delta_{n}^{\varepsilon}=f\left(T^{n} x\right)-f\left(T_{\omega}^{n} x\right)$. In fact both $T$ and $T_{\omega}$ are 510 affected by the arithmetic roundoff, which in a single step 511 introduces an error of order $\varepsilon_{\text {num }}$. The orbit $T^{n}$ is replaced 512 by the perturbed orbit $T_{\omega^{\prime}}^{n}$, where $\omega_{l}^{\prime}$ are now "random" 513 variables in the space, say, $\Omega_{\varepsilon_{\text {num }}}(0)$ around the unperturbed 514 component 0 . In the same way the random orbit $T_{\omega}^{n}$ will be 515 replaced by a new random orbit $T_{\omega^{\prime \prime}}^{n}$, where the components 516 $\omega_{l}^{\prime \prime}$ are now random variables in the space $\Omega_{\varepsilon_{\text {num }}}\left(\omega_{l}\right)$ around 517 the component $\omega_{l}$. Comparing $\Delta_{n}^{\varepsilon, \varepsilon_{\mathrm{num}}}=f\left(T_{\boldsymbol{\omega}^{\prime}}^{n} x\right)-f\left(T_{\boldsymbol{\omega}^{\prime \prime}}^{n} x\right) 518$ with the analytical estimate for $\Delta_{n}^{\varepsilon}$, or just letting $\varepsilon_{\text {num }} \rightarrow 0519$ by using arbitrary precision arithmetic, we can check that if $\mathbf{5 2 0}$ $\varepsilon \gg \varepsilon_{\text {num }}$, there is a time scale relevant for the study of $\Delta_{n}^{\varepsilon}, \mathbf{5 2 1}$ below which the additional error $\Delta_{n}^{\varepsilon}-\Delta_{n}^{\varepsilon, \varepsilon_{\mathrm{num}}}$ is negligible 522 with respect to $\Delta_{n}^{\varepsilon}$. Since the numerical roundoff error in 523 double precision for a variable on the torus is of order 524 $\varepsilon_{\text {num }} \approx 10^{-16}$, we are going to use additive noise $\varepsilon>10^{-9} .525$ The choice $\varepsilon \approx 10^{-8}$, corresponding to single precision nu- 526 merical roundoff, is of interest for future investigations on 527 the statistics of roundoff errors in the iteration of maps. The $\mathbf{5 2 8}$ convergence in distribution of the process $\Delta_{n}^{\varepsilon, \widetilde{\varepsilon}}$ is guaranteed 529 by the generalization of Theorem 1 provided in Remark 1. We 530 observe that Sauer in Ref. 13 faced the same problem in 531 developing a theory of true and perturbed orbits while 532 checking the predictions of computer experiments. He wrote, 533 "We computed the trajectory average in high precision (used 534 to represent the "true" value) for a long trajectory and com- 535 pared it with the trajectory computed with one-step random 536 error of size $\varepsilon . "$

537

\section{A. Hénon map}

538

The Hénon map is a 2D map defined by 539 


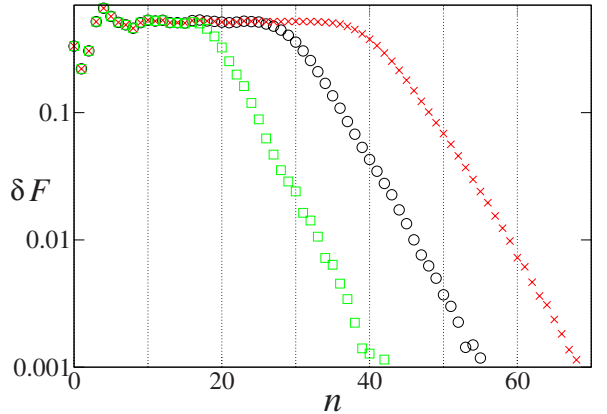

FIG. 7. (Color online) Decay of fidelity for the Hénon map, Monte Carlo integration with $N=10^{7}$. Green squares: $\varepsilon=10^{-4}$. Black circles: $\varepsilon=10^{-6}$. Red crosses: $\varepsilon=10^{-8}$.

$540 T \mathbf{x}=\left\{\begin{array}{l}x^{\prime}=y+1-a x^{2} \\ y^{\prime}=b x\end{array}\right.$

541 We consider the canonical values $a=1.4$ and $b=0.3$, for 542 which the map is known to be chaotic, at least numerically. 543 We remind that Benedicks and Carleson ${ }^{14}$ proved that there 544 exists a set of positive Lebesgue measure $S$ in the parameter 545 space such that the Hénon map has a strange attractor when546 ever $(a, b) \in S$. The value of $b$ is very small and the attractor 547 lives in a small neighborhood of the $x$-axis. For those values 548 of $a$ and $b$, one can prove the existence of the physical mea549 sure and of a stationary measure under additive noise, which 550 is supported in the basin of attraction and that converges to 551 the physical measure in the zero noise limit. ${ }^{15}$ It is still un552 known whether such results could be extended to the "his553 torical" values that we consider here.

554 The orbit starting at $\left(x_{0}, y_{0}\right)$ will either approach the 555 Hénon strange attractor or diverge to infinity. We study a 556 connected subset $D$ included in the basin of the attractor and 557 define on it the perturbed map

558

$$
T_{\varepsilon \xi} \mathbf{x}=\left\{\begin{array}{l}
x^{\prime}=y+1-a x^{2}+\varepsilon \xi_{x} \\
y^{\prime}=b x+\varepsilon \xi_{y} .
\end{array}\right.
$$

559 We have verified that using $D=(x, y)$ with $-1<x<1$ and $560-1 / 2<y<1 / 4$ the set $D$ is stable under small perturbations 561 [i.e., the point $\left(x_{0}, y_{0}\right)$ does not diverge to infinity under $T_{\varepsilon}$ 562 for small enough $\varepsilon$ ].

563 We studied the decay of fidelity [Eq. (24)] with observ564 ables $x$ and $y$. After a transient that depends on the definition 565 of the set $D$, the fidelity reaches a plateau, and then follows 566 a decay phase (Fig. 7), whose rate does not depend on $\varepsilon$. The

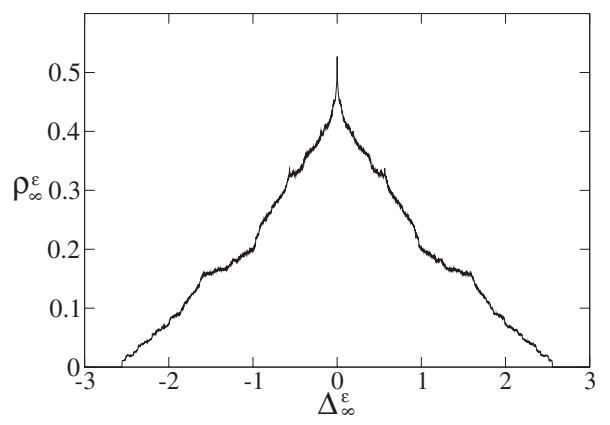

FIG. 9. $\rho_{\infty}^{\varepsilon}$ for the Hénon map with $\varepsilon=10^{-8}, f(\mathbf{x})=x$ given by the average of $10^{6}$ samplings of $\rho_{n}^{\varepsilon}$, each one obtained using a Monte Carlo method with $10^{3}$ starting points $\left(N=10^{9}\right)$ and $\Delta x=10^{-3}$.

$\varepsilon$ dependence is manifest in the outset of the decay (end of 567 plateau). We name the value of $n$ at which the decay phase 568 starts $n_{*}$ since its $\varepsilon$ dependence is qualitatively compatible 569 with Eq. (20). The height of the plateau, $n_{*}$, and the decay 570 law are independent of the choice of $D$, as expected due to 571 the ergodic properties of the map. The behavior of the $y 572$ observable is almost the same as that of $x$. We successfully 573 checked (Fig. 8) that the transition from $\rho_{0}^{\varepsilon}=\delta(x)$ to the 574 asymptotic $\rho_{\infty}^{\varepsilon}$ starts with the outset of the decay phase of the 575 fidelity (for example, around $n_{*} \approx 35$ in Fig. 7 for $\varepsilon=10^{-8}$ ). 576 Below this time scale the error distribution function can be 577 approximated by a $\delta$ function and thus the perturbed system 578 can be considered as equivalent to the unperturbed one. We 579 finally averaged $\rho_{n}^{\varepsilon}$ over several $n>n_{*}$ in order to obtain $\rho_{\infty}^{\varepsilon} 580$ (Fig. 9) and checked, with good results, the validity of 581 Eq. (2).

\section{B. Baker's map}

The Baker's map is defined by $T \mathbf{x}=\mathbf{x}^{\prime}$, where

$x^{\prime}= \begin{cases}\gamma_{a} x \bmod 1 & \text { if } y<\alpha \\ \frac{1}{2}+\gamma_{b} x \bmod 1 & \text { if } y \geq \alpha,\end{cases}$

$$
y^{\prime}= \begin{cases}\frac{y}{\alpha} \bmod 1 & \text { if } y<\alpha \\ \frac{y-\alpha}{1-\alpha} \bmod 1 & \text { if } y \geq \alpha,\end{cases}
$$

while its perturbed version is
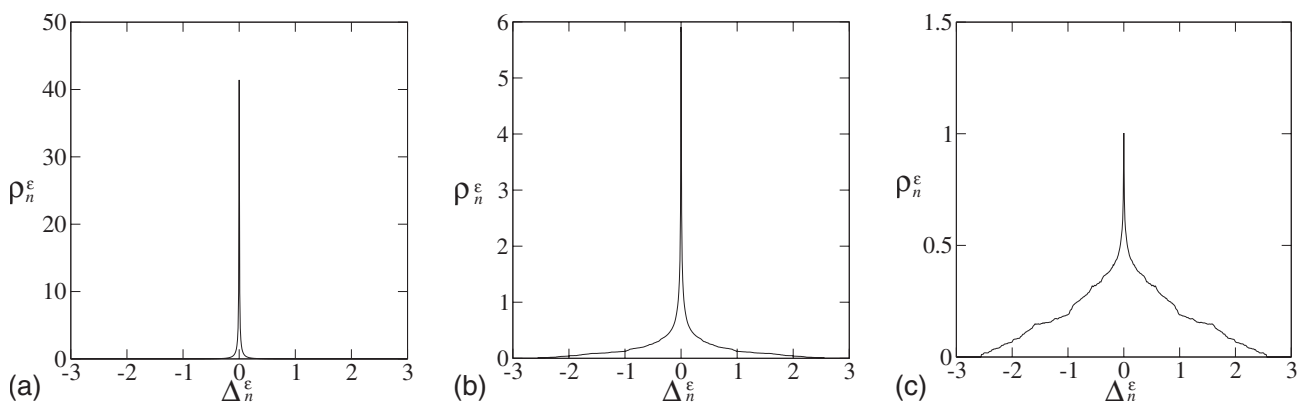

FIG. 8. $\rho_{n}^{\varepsilon}$ for the Hénon map, $\varepsilon=10^{-8}, f(\mathbf{x})=x$. (a) $n=34$; (b) $n=44$; (c) $n=54$. Monte Carlo sampling using $N=10^{8}$ initial points and space discretization $\Delta x=10^{-2}$. 

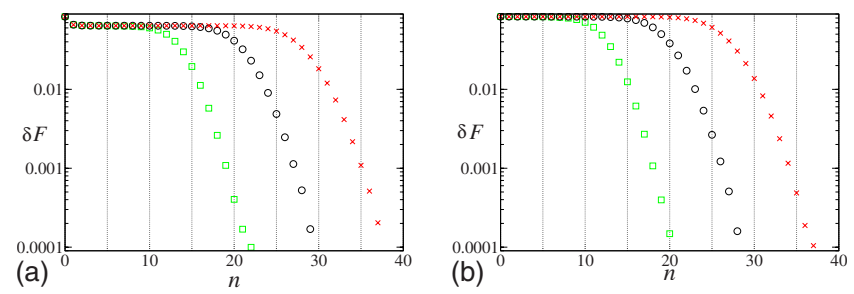

FIG. 10. (Color online) Decay of fidelity for the Baker's map, Monte Carlo integration with $N=10^{7}$. Green squares: $\varepsilon=10^{-4}$. Black circles: $\varepsilon=10^{-6}$. Red crosses: $\varepsilon=10^{-8}$. (a) $x$ observable. (b) $y$ observable.

588

$$
T_{\varepsilon \xi} \mathbf{X}=\left(x^{\prime}+\varepsilon \xi_{x} \bmod 1, y^{\prime}+\varepsilon \xi_{y} \bmod 1\right) .
$$

589 Using $\gamma_{a}=\frac{1}{5}, \gamma_{b}=\frac{1}{4}$, and $\alpha=\frac{1}{3}$ we studied the decay of fidelity 590 for the $x$ and $y$ observables (Fig. 10).

591 While the decay of fidelity for $\psi(\mathbf{x})=y$ shows a plateau 592 and then a decay phase, for $\psi(\mathbf{x})=x$ the plateau is preceded 593 by a decrease in the value of fidelity due to the convergence 594 to the invariant measure. The difference between the first 595 steps of fidelity for $y$ and $x$ is due to the different nature of 596 the invariant distributions (uniform for $y$ and a Cantor set for $597 x$ ); nevertheless the outset of fidelity decay (end of plateau) 598 happens roughly at the same $n_{*}$ for the two observables $599\left(n_{*} \approx 20\right.$ for $\left.\varepsilon=10^{-8}\right)$. Once again the $\varepsilon$ dependence of $n_{*}$ is 600 in agreement with Eq. (20), and it is followed by an $\varepsilon$ inde601 pendent decay law. Also for this map the analysis of fidelity 602 predicts quite well the outset of the transient from a delta 603 function to the asymptotic distribution for the error distribu604 tion (not shown). While for $f(\mathbf{x})=y, \rho_{\infty}^{\varepsilon}$ is simply the trian605 gular function, for the observable $x$ we have a Cantor struc606 ture that can be better appreciated increasing the precision of 607 the computation (Fig. 11). We also checked that Eq. (2) holds 608 for this system. In order to understand the irregular shape of 609 the pdf for the observable $x$, we modify the Baker's map in 610 such a way to get the usual ternary Cantor set on the $x$-axis. 611 This can be easily achieved by writing the modified Baker's 612 map as

10

$$
x^{\prime}= \begin{cases}\frac{x}{3} & \text { if } y<\frac{1}{2} \\ \frac{1}{2}+\frac{x}{3} & \text { if } y \geq \frac{1}{2},\end{cases}
$$

614
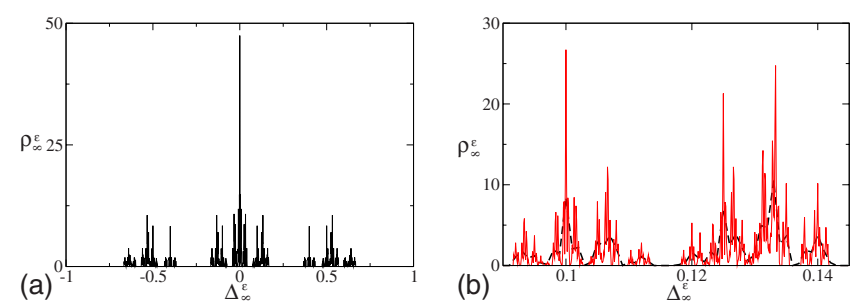

FIG. 11. (Color online) (a) $\rho_{\infty}^{\varepsilon}$ for the Baker's map, $\varepsilon=10^{-8}, x$ observable. Monte Carlo sampling using $N=10^{9}$ and $\Delta x=10^{-3}$. (b) detail of $\rho_{\infty}^{\varepsilon}$ for the Baker's map, $\varepsilon=10^{-8}, x$ observable. Black, dashed: Monte Carlo using $N$ $=10^{9}$ and $\Delta x=10^{-3}$. Red, continuous: Monte Carlo using $N=10^{10}$ and $\Delta x$ $=10^{-4}$.
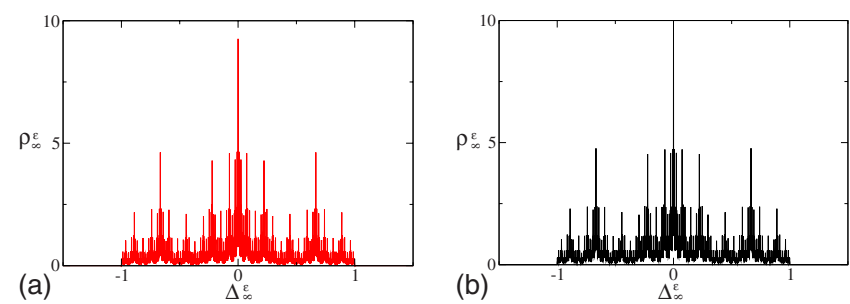

FIG. 12. (Color online) $\rho_{\infty}^{\varepsilon}$ for the modified Baker map (whose invariant measure corresponds to a ternary Cantor set) as obtained by Eq. (28) (a) and by Monte Carlo method (b).

$$
y^{\prime}= \begin{cases}2 y & \text { if } y<\frac{1}{2} \\ 2 y-1 & \text { if } y \geq \frac{1}{2} .\end{cases}
$$

The physical SRB measure will be the direct product of an 616 absolutely continuous measure along the $y$-axis and the sin- 617 gular Cantorian measure along the $x$-axis. ${ }^{16}$ The latter is con- 618 structed as the weak limit of a sequence of measures which 619 give equal weight $2^{-n}$ to the $2^{n}$ closed intervals of length $3^{-n} 620$ which contain the Cantor set for all $n$. The Fourier transform 621 of this measure, say, $\mu_{x}$, is known. ${ }^{17}$ If we consider the ob- 622 servable $x$, the characteristic function of the asymptotic error 623 will be $\int e^{i x u} d \mu_{x} \overline{\int e^{i x u} d \mu_{\varepsilon}}$. Let us suppose that the stationary 624 measure is close to the SRB measure, which is the case since 625 the Baker's transformation is uniformly hyperbolic, belongs 626 to the class $\mathcal{M}$, and is stochastically stable. Therefore for 627 small $\varepsilon$ we could assume that the second integral in the pre- 628 ceding product is taken with respect to $\mu_{x}$, and thus our 629 characteristic function is $\varphi_{\infty}(u)=\left|\int e^{i u x} d \mu_{x}\right|^{2}$. The latter can 630 be computed: Zygmund ${ }^{17}$ gives (see also Ref. 18 for a 631 former study of this quantity)

632

$$
\varphi_{\infty}(u)=\prod_{k=1}^{\infty} \cos ^{2}\left[u 3^{-k}\right] .
$$

Zygmund also proved that $\varphi_{\infty}(u)$ is not summable and we 634 recall that this is a sufficient condition in order to get the 635 inversion formula (11). Despite this, we compute numeri- 636 cally the improper integral transform (11) and we get an 637 excellent agreement with the numerical issues (Fig. 12). We 638 outline that even if for any spatial discretization $\Delta x$ we can 639 numerically compute, obtaining the same result, the distribu- 640 tion function $\rho_{\varepsilon}^{n}$ with three different methods [Monte Carlo 641 sampling of errors falling in the interval $[x, x+\Delta x]$, inverse 642 Fourier transform of Eq. (2), and inverse Fourier transform 643 of Eq. (28)], we are not sure that the process converges in the 644 $\Delta x \rightarrow 0$ limit, since the inverse Fourier transform could be ill 645 defined (see Appendix D and the analogous discussion in 646 Sec. IV C). Figure 11 shows clearly that at the numerically 647 accessible $\Delta x$ values the process still has not converged. 648

\section{Intermittent map}

The intermittent map on the torus is defined as 650

$$
T_{\alpha} x= \begin{cases}x+2^{\alpha} x^{\alpha+1} \bmod 1 & \text { if } 0 \leq x<\frac{1}{2} \\ x-2^{\alpha}(1-x)^{\alpha+1} \bmod 1 & \text { if } \frac{1}{2} \leq x<1\end{cases}
$$

with $0<\alpha<1$. It has been proved by several authors that the 652 decay of correlations follows a power law as $n^{1-1 / \alpha} \cdot{ }^{19-21}$ This 653 


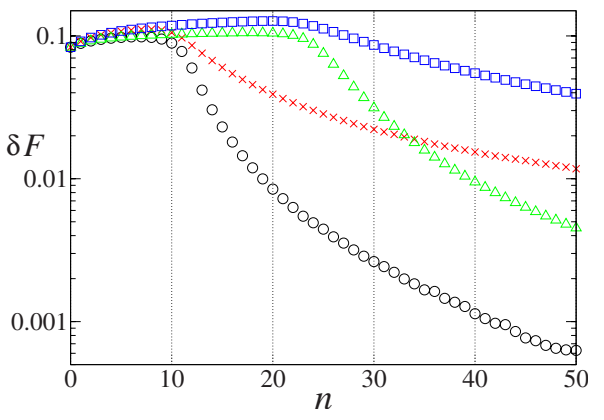

FIG. 13. (Color online) Decay of fidelity for the intermittent map, Monte Carlo integration with $N=10^{7}$. Black circles: $\varepsilon=10^{-4}, \alpha=0.5$. Red crosses: $\varepsilon=10^{-4}, \alpha=0.99$. Green triangles: $\varepsilon=10^{-8}, \alpha=0.5$. Blue squares: $\varepsilon=10^{-8}$, $\alpha=0.99$.

654 rate is optimal in the sense that it gives also a lower bound 655 for a large class of observables vanishing in a neighborhood 656 of the neutral fixed point. ${ }^{22,23}$ The density $h$ of the absolutely 657 continuous invariant measure has a singularity in the neigh658 borhood of zero of the type $x^{-\alpha}$. The density $h_{\varepsilon}$ of the sta659 tionary measure verifies the $\mathcal{L}_{m}^{1}$ estimate: $\left\|h-h_{\varepsilon}\right\|_{\mathcal{L}_{m}^{1}} \sim \varepsilon^{1-\alpha}{ }^{24}$ $660 \mathrm{It}$ is interesting to remark that the problem of the stochastic 661 stability for this map is not fully understood. The papers ${ }^{25,26}$ 662 prove the weak convergence of the absolutely continuous 663 stationary measure (under the additive noise) to a convex 664 combination of the absolutely continuous invariant measure 665 and of the Dirac mass at the neutral fixed point. The presence 666 of this atomic measure in the weak limit is questionable; it 667 prevents also any kind of strong convergence (in the $\mathcal{L}_{m}^{1}$ 668 sense).

669 Our Monte Carlo integrations for the decay of correla670 tions are compatible with the analytically predicted power 671 law, even if an exact computation of the exponent of the 672 power law is not feasible to a numerical analysis since the 673 convergence to the predicted law is slow in $n$. The decay of 674 fidelity for this map shows a growing phase (that depends on $675 \alpha$ and is due to the convergence to the asymptotic measure), 676 an $\alpha$ independent outset of decay $n_{*}(\varepsilon)$ (end of "plateau") 677 whose $\varepsilon$ dependence is in qualitative agreement with Eq. 678 (20), followed by an $\alpha, \varepsilon$ dependent decay law (Fig. 13). Our 679 results show that in the $\varepsilon \rightarrow 0$ limit the decay law is compat680 ible with a power law (the exponent seems to be higher than 681 the one of the decay of correlations), while for high values of $682 \varepsilon$ the decay law appears to be at least exponential. As a guess 683 we claim a decay law $n^{-b} f(\varepsilon, n)$, where $f(\varepsilon, n)$ should be 684 bounded by the decay law for translation and identity, Eq. 685 (16). We checked that $n_{*}$ corresponds to the beginning of the 686 transition from the delta function to the asymptotic error dis687 tribution function (not shown) and that Eq. (2) holds for $\rho_{\infty}^{\varepsilon}$ 688 (which is shown in Fig. 14). We believe that the proposed 689 decay law is also satisfied by the annealed random correla690 tion integral given by the left-hand side of Eq. (6): this is a 691 nice theoretical challenge.

692 Equation (D1) predicts a divergence for the asymptotic 693 distribution $\rho_{\infty}^{\varepsilon}$ in 0 whenever $\alpha>1 / 2$. We have indeed veri694 fied that the height of $\rho_{\infty}^{\varepsilon}(0)$, numerically computed using a

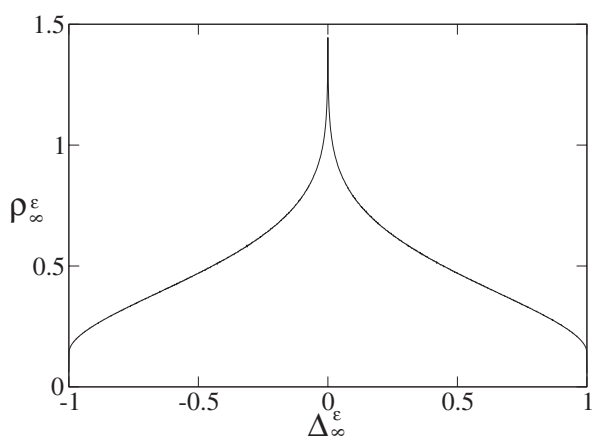

FIG. 14. $\rho_{\infty}^{\varepsilon}$ for the intermittent map, $\alpha=0.5, \varepsilon=10^{-8}$. Monte Carlo sampling using $N=10^{9}$ and $\Delta x=10^{-3}$.

Monte Carlo sampling with space discretization $\Delta x$, grows 695 for the numerically accessible values of $\Delta x$, at least as 696 $\log (\Delta x)$.

\section{Quadratic map}

We consider in this section the quadratic map $T_{a} x=a 699$ $-x^{2}, a>0$. The image under $T_{a}$ of $[-\sqrt{2 a}, \sqrt{2 a}]$ is $[-a, a]$ and 700 thus for $a<2$ we can define the perturbed map $T_{a \varepsilon \xi} x=a 701$ $-x^{2}+\varepsilon \xi$ from $[-\sqrt{2 a}, \sqrt{2 a}]$ to itself provided that $\varepsilon<\sqrt{2 a}-a .702$

This class of maps has been investigated in Ref. 27 from 703 the point of view of stochastic stability. First of all the au- 704 thors restricted the choice of the parameter $a$ to the set of 705 positive measure for which there exists a unique absolutely 706 continuous invariant measure with density $h$ (Jakobson's 707 theorem $^{28}$ ). For those values, the map satisfies also the 708 Benedicks-Carleson ${ }^{14}$ conditions which were important for 709 establishing the other perturbative results under the additive $\mathbf{7 1 0}$ noise, namely, (i) the existence of an absolutely continuous 711 stationary measure with density $h_{\varepsilon}$ which converges in the 712 $\mathcal{L}_{m}^{1}$ norm to $h$ (stochastic stability) and (ii) the existence of 713 exponential decay of correlations for the unperturbed map 714 and for the perturbed system with decay rates which are uni- 715 formly bounded in the noise (provided $\varepsilon$ is small enough), as 716 in formulas (5) and (6). The latter (see Ref. 9) allows us to 717 apply Theorem 1 on the fidelity so that the estimate (12) for 718 the rate of convergence to the characteristic function of the 719 asymptotic error holds. We verified, through a bifurcation 720 analysis, that in a neighborhood of $a=1.6$ the map is chaotic, 721 and thus we have used this value for our investigation. 722

After a transient (which is due to the difference between 723 the uniform distribution we use as initial condition for our 724 Monte Carlo method and the invariant measure of the map) 725 there is a plateau that ends at $n_{*}$, whose $\varepsilon$ dependence is in 726 qualitative agreement with Eq. (20), Fig. 15. To $n_{*}$ corre- 727 sponds the beginning of the transition of the error distribu- 728 tion function (not shown). The asymptotic error distribution 729 is shown in Fig. 16 [we have verified that the same result can 730 also be obtained using Eq. (2)].

\section{E. Standard map}

We study the standard map defined as 


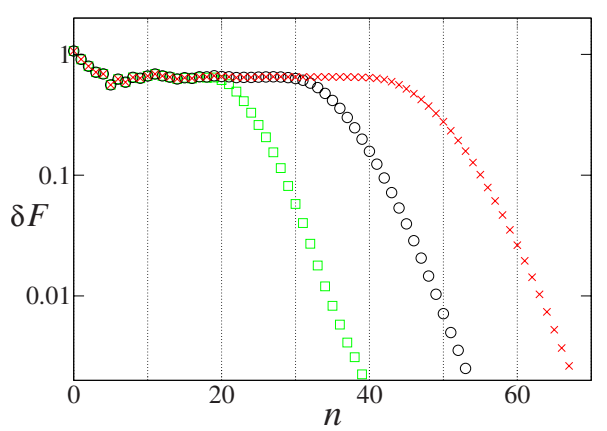

FIG. 15. (Color online) Decay of fidelity for the quadratic map, $a=1.6$, Monte Carlo integration with $N=10^{8}$. Green squares: $\varepsilon=10^{-4}$. Black circles: $\varepsilon=10^{-6}$. Red crosses: $\varepsilon=10^{-8}$.

$734 T \mathbf{x}=\left\{\begin{array}{l}y^{\prime}=y+K \sin (x) \\ x^{\prime}=x+y^{\prime} .\end{array}\right.$

735 We analyze its behavior considering three values of $K: K=10$ 736 (chaotic behavior), $K=10^{-2}$ (regular behavior), and $K=2$ 737 (mixed behavior). We recall that the breakup of the last in738 variant curve corresponding to the golden mean frequency 739 occurs for $K \simeq 1$.

740 Case $K=10$ : The chaotic case can be easily understood 741 using the framework developed for other maps. For the de742 cay of fidelity we have a plateau, followed by an outset of 743 decay that occurs at $n_{*}$ in qualitative agreement with Eq. (20) 744 [(a) of Fig. 17]. $n_{*}$ corresponds to the beginning of the tran745 sient of the error function. The asymptotic error function $\rho_{\infty}^{\varepsilon}$ 746 is triangular and Eq. (2) applies.

747 Case $K=10^{-2}$ : In the regular regime there is no clear 748 outset of decay, but just an $\varepsilon$ dependent decay law [(b) of 749 Fig. 17]. The asymptotic error function is reached when the 750 fidelity reaches a value of a few orders of magnitude lower 751 than its initial value. The asymptotic error function is trian752 gular and Eq. (2) holds. The decay for $y$ follows the same 753 law as rotations on the circle, while the decay for $x$ is 754 quicker. This can be easily understood by considering the 755 map where $K \sin (x)$ is replaced by $K \xi$, where $\xi$ is a random 756 variable in $[-1,1]$. In the continuum limit $K \rightarrow 0$, see Appen757 dix $\mathrm{C}$, we have a diffusive process with Gaussian distribution 758 where the mean square deviation is $K^{2} n$ for the $y$ variable, $759 K^{2} n^{3}$ for the $x$ variable proving that the relaxation for the 760 latter is faster.

761 Case $K=2$ : For $K=2$ the system exhibits features of both

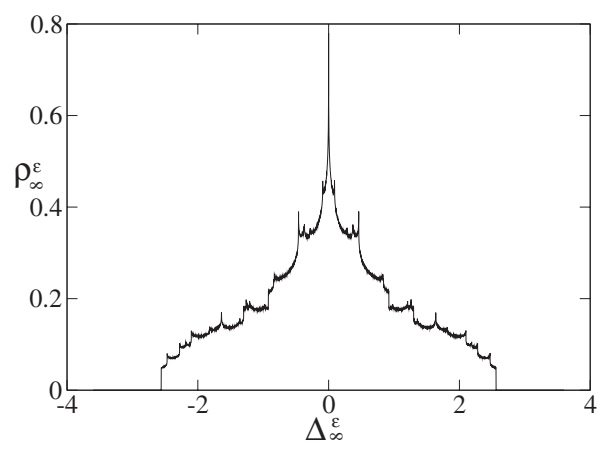

FIG. 16. $\rho_{\infty}^{\varepsilon}$ for the quadratic map, $a=1.6, \varepsilon=10^{-8}$. Monte Carlo sampling using $N=10^{9}$ and $\Delta x=10^{-3}$.
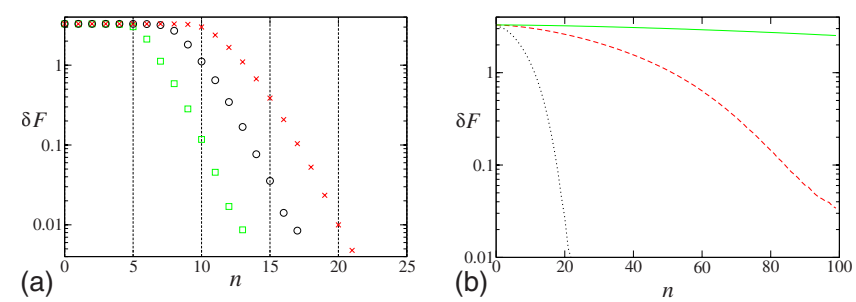

FIG. 17. (Color online) Decay of fidelity for the standard map, $x$ observable, Monte Carlo integration with $N=10^{7}$. (a) $K=10$ (green squares: $\varepsilon=10^{-4}$; black circles: $\varepsilon=10^{-6}$; red crosses: $\varepsilon=10^{-8}$ ). (b) $K=10^{-2}$ (black, dotted: $\varepsilon$ $=10^{-1}$; red, dashed: $\varepsilon=10^{-2}$; green: $\varepsilon=10^{-3}$ ).

regular and chaotic maps. The decay of fidelity presents a 762 plateau ending at $n_{*}$ in agreement with Eq. (20). This phase 763 is followed by a quick, almost $\varepsilon$ independent decay phase, 764 and then by a slower $\varepsilon$ dependent phase (Fig. 18). After the 765 first quick decay phase we reach an asymptotic error function 766 for the chaotic component only, while if $\varepsilon$ is low enough, we 767 still have a $\delta$ function for the regular component (Fig. 19). 768 For low values of $\varepsilon$ we can consider this as a "metastable 769 error distribution function" since it does not change during a 770 time scale much longer than the decay of the chaotic com- 771 ponent. The study of the $\varepsilon$ dependent decay phase of the 772 regular component in a map with mixed behavior is more 773 difficult than for a regular map, probably due to the interac- 774 tion between the chaotic and regular components. Indeed, for 775 purely chaotic and regular maps we found that the decay of 776 fidelity was almost independent of the function $\psi$, and thus 777 we limited ourselves to the study of the less computationally 778 expensive $\psi(\mathbf{x})=x$. But for the standard map with $K=2$ even 779 when the fidelity for $\psi(\mathbf{x})=x$ has reached a value of a few 780 orders of magnitude lower than its initial value, the error 781 function has not reached its asymptotic value (triangular dis- 782 tribution). Since the error distribution $\rho_{\varepsilon}^{n}$ is given by the Fou- 783 rier transform of $\varphi_{\varepsilon}^{n}(u)$, i.e., by the fidelity computed using 784 $\psi_{1}=\psi_{2}^{*}=e^{i u x}$, the time scales of the transient of $\rho_{\varepsilon}^{n}$ are deter- 785 mined by the decay of fidelity of $e^{i u x}$. While for all the pre- 786 viously studied systems the decay of fidelity for $e^{i u x}$ resulted 787 to be $u$ independent, and thus the system had a single decay 788 time scale, for the standard map with $K=2$ the decay of $e^{i u x} 789$ results to be strongly $u$ dependent (see Fig. 20; the change in 790 slope around $n \approx 50$ corresponds to the end of the decay of 791 the chaotic component and the beginning of the "metastable" 792 phase, which is quite short for $\varepsilon=10^{-1}$ but considerably 793

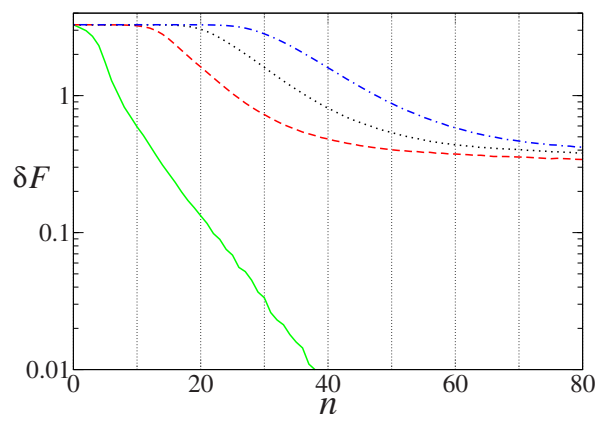

FIG. 18. (Color online) Decay of fidelity for the standard map, $x$ observable, $K=2$, Monte Carlo integration with $N=10^{7}$. Green: $\varepsilon=10^{-1}$. Red, dashed: $\varepsilon=10^{-4}$. Black, dotted: $\varepsilon=10^{-6}$. Blue, dot-dashed: $\varepsilon=10^{-8}$. 

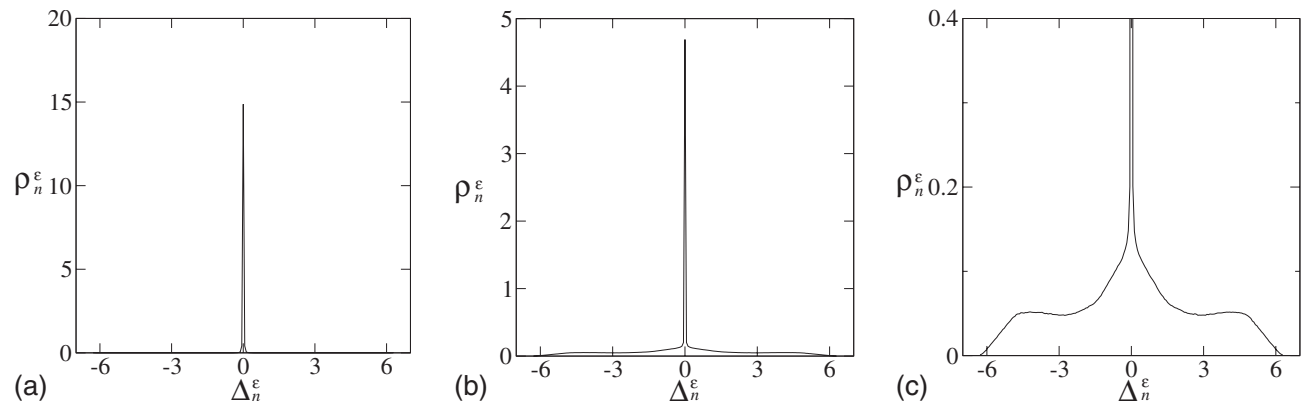

FIG. 19. $\rho_{n}^{\varepsilon}$ for the standard map, $K=2, \varepsilon=10^{-4}$, $x$ observable. (a) $n=9$; (b) $n=49$; (c) detail of the metastable distribution $\left(\rho_{49}\right)$. Monte Carlo sampling using $N=10^{7}$ and $\Delta x=\pi / 50$.

794 longer for lower values of $\varepsilon$ ), and thus the regular component 795 of a map with mixed behavior has not a single decay scale. 796 We have verified that when the fidelity computed using $e^{i x}$, 797 which, on the basis of our numerical tests, has probably the 798 longest decay time, reaches a value of $\approx 10^{-3}$, we have the 799 convergence to the triangular asymptotic error distribution $800 \rho_{\infty}^{\varepsilon}$.

\section{V. CONCLUSIONS}

802 We have analyzed the statistical properties of the error 803 introduced by an additive random noise in a discrete dynami804 cal system by comparing the distributions obtained from 805 Monte Carlo computations and from the fidelity, which, for a 806 suitable choice of the observables, provides the Fourier 807 transform of the pdf of the error. This pdf is obtained in the 808 limit of large iteration time and the rate of this convergence 809 is very important to discriminate between regular and chaotic 810 motions. For rotations and Bernoulli maps an exact result 811 and an optimal estimate for the convergence rate are ob812 tained. The exponential convergence rate for regular maps 813 and the superexponential one for chaotic maps appear to be 814 quite general as we indicate by several numerical examples. 815 The initial distribution of errors is a Dirac $\delta$ function with 816 support at zero, whereas the asymptotic distribution depends 817 on the invariant and the stationary measures. When both are 818 Lebesgue the asymptotic distribution is triangular as for two 819 points randomly chosen on an interval. For measures abso820 lutely continuous with respect to Lebesgue the asymptotic 821 distribution is smooth, whereas it is spiky for singular con822 tinuous measures typical of attractors with a Cantorian struc-

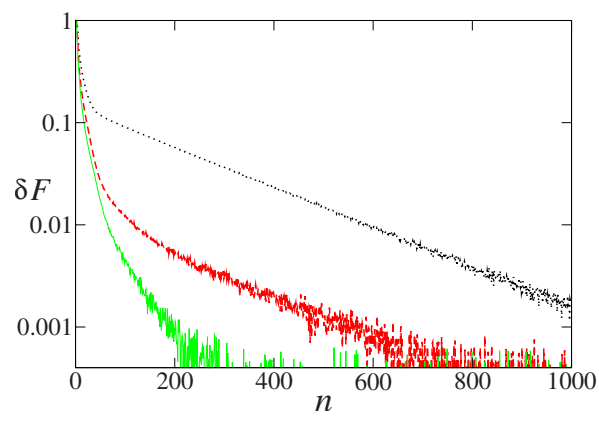

FIG. 20. (Color online) Decay of fidelity for the standard map, $K=2, \varepsilon$ $=10^{-1}$, Monte Carlo integration with $N=10^{7}$. Black, dotted: $\psi_{1}=\psi_{2}^{*}=e^{i x}$. Red, dashed: $\psi_{1}=\psi_{2}^{*}=e^{i 2 x}$. Green: $\psi_{1}=\psi_{2}^{*}=e^{i 3 x}$. ture (fractals). As we said above, the ergodic properties of $\mathbf{8 2 3}$ the map are reflected in the way the asymptotic limit is $\mathbf{8 2 4}$ reached: the exponential and superexponential convergence $\mathbf{8 2 5}$ rates are the same as for a random walk when the determin- $\mathbf{8 2 6}$ istic evolution is linear or exponential as it can be easily 827 proved in the continuum limit. Whereas the asymptotic limit $\mathbf{8 2 8}$ is reached smoothly for regular maps, for the chaotic ones 829 there is a sharp transition whose location depends only on $\mathbf{8 3 0}$ the perturbation strength. For maps with chaotic and regular 831 invariant regions, such as the standard map short after the $\mathbf{8 3 2}$ breakup of the last invariant curve, there is first a sharp tran- $\mathbf{8 3 3}$ sition followed by a smooth decay. Finally the procedure we 834 outlined applies to the analysis of roundoff errors for the $\mathbf{8 3 5}$ numerical maps, a key issue in the numerical analysis of $\mathbf{8 3 6}$ dynamical systems. We have already applied the procedure 837 developed in this paper to analyze the roundoff errors and the 838 results will be soon submitted for publication. We can antici- 839 pate that the global error grows linearly for regular maps and $\mathbf{8 4 0}$ exponentially for chaotic maps, while the asymptotic error $\mathbf{8 4 1}$ distribution reflects the properties of the invariant measure. 842 The convergence rate of fidelity can be quite different from 843 the one observed for additive noise due to the deterministic 844 nature of roundoff, nevertheless by increasing the complexity 845 of the map the "pseudorandom" character of roundoff errors 846 becomes more pronounced, and the decay law becomes, in 847 particular for chaotic maps, equivalent to that of maps per- 848 turbed with additive noise. To conclude we claim that we 849 have provided a reliable and constructive tool to investigate 850 analytically and numerically the perturbations induced by 851 random errors. This procedure can be applied also to numeri- 852 cal roundoff errors even though in that case no rigorous gen- 853 eral results can be formulated because the algorithms imple- 854 mented in finite precision arithmetic are hardware dependent. 855

\section{ACKNOWLEDGMENTS}

F.Z. has been partially supported by the GDRE 224857 GREFI-MEFI (jointly sponsored by the French CNRS and 858 the Italian INDAM). The authors thank the anonymous ref- 859 erees whose remarks and suggestions contributed to improve $\mathbf{8 6 0}$ the paper. 


\section{APPENDIX A: COMPARISON TO SOME RESULTS 863 BY SAUER}

864 We now compare our results with the work of Sauer in 865 Ref. 13. He considered a smooth observable $g$ on the phase 866 space $X$ and the algebraic difference

867

$$
\Delta(g) \equiv\langle g\rangle_{\text {computed }}-\langle g\rangle_{\text {true }},
$$

868 where $\langle g\rangle_{\text {true }}$ is the ergodic average computed along the true 869 trajectory of $T$ and $\langle g\rangle_{\text {computed }}$ is the ergodic average com870 puted along the perturbed orbit with noise size $\varepsilon$. Sauer pro871 posed the following heuristic scaling:

$872 \Delta(g)=K \varepsilon^{h}$,

873 where $K$ is a constant and $h$ is an "exponent expressing the 874 severity of the fluctuations of the Lyapunov exponent."13

875 For a map belonging to the class $\mathcal{M}$, we simply have $876\langle g\rangle_{\text {true }}=\int_{X} g d \mu$. The ergodic average for the random pertur877 bations introduced in Sec. II C will be

878

$$
\lim _{n \rightarrow \infty} \frac{1}{n} \sum_{l=0}^{n-1} g\left(T_{\omega_{l}} T_{\omega_{l-1}} \cdots T_{\omega_{0}} x\right) .
$$

879 For the maps in $\mathcal{M}$ the stationary measure is ergodic and this 880 means that the previous Birkhoff sum goes to $\int_{X} g d \mu_{\varepsilon}$, for $x$ 881 chosen $m$-almost everywhere and for almost all the realiza882 tions $\boldsymbol{\omega}$ taken with respect to the measure $\theta_{\varepsilon}^{\mathbb{N}}$; see, for in883 stance, Ref. 29. It is therefore reasonable to put $\langle g\rangle_{\text {computed }}$ $884=\int_{X} g d \mu_{\varepsilon}$ in such a way that

885

$$
\Delta(g)=\int_{X} g d \mu_{\varepsilon}-\int_{X} g d \mu
$$

886 [compare with (i) in Remark 2]. Now, let us suppose that the 887 map $T \in \mathcal{M}$ has an invariant measure $\mu$ which is absolutely 888 continuous with respect to the Lebesgue measure $m$ with 889 density $h$; we suppose also that the stationary measure $\mu_{\varepsilon}$ is 890 absolutely continuous with density $h_{\varepsilon}$. The systems in $\mathcal{M}$ 891 usually enjoy the strong stochastic stability property which 892 means the convergence of $h_{\varepsilon}$ to $h$ in the $\mathcal{L}_{m}^{1}$ norm. The 893 paper $^{24}$ quotes several examples where this $\mathcal{L}_{m}^{1}$ convergence 894 is explicitly computed for additive noise, with rigorous argu895 ments or numerically (even for systems outside $\mathcal{M}$ ), as a 896 function of the noise size $\varepsilon$; the scalings found are of the 897 type

898

$$
\left\|h-h_{\varepsilon}\right\|_{\mathcal{L}_{m}^{1}} \leq \text { const } \varepsilon^{\gamma}
$$

899 where $\gamma$ is an exponent depending upon the map T. This 900 exponent seems related to the smoothness of the map rather 901 than to the Lyapunov exponent. For example, for continuous 902 uniformly expanding maps of the circle $\gamma=2$, while $\gamma=1$ if 903 the map has discontinuities and it is therefore piecewise ex904 panding. For Misiurewicz quadratic maps (see also Sec. 905 IV D), $\gamma=0.5$ and it ranges between 0.3 and 0.7 for the in906 termittent map investigated in Sect. IV C. We have also seen 907 that there are examples for which the invariant and the sta908 tionary measure are the same, $h=h_{\varepsilon}$, but the pdf of the 909 asymptotic error for the observable identity $f(x)=x$ is a 910 smooth function whose support has diameter twice the sup911 port of the invariant measure.
Since, by Hölder inequality,

$$
|\Delta(g)| \leq\|g\|_{C^{0}}\left\|h-h_{\varepsilon}\right\|_{\mathcal{L}_{m}^{1}}\|g\|_{C^{0}} \text { const } \varepsilon^{\gamma},
$$

the comparison with the previous bound claimed by Sauer is 914 not obvious especially for the meaning of the scaling expo- 915 nent of $\varepsilon$. One possible explanation, which deserves better 916 investigation, is that the fluctuations of the Lyapunov expo- 917 nent play a role whenever we replace $\langle g\rangle_{\text {computed }}$ with the 918 Birkhoff sum $1 / n \sum_{l=0}^{n-1} g\left(T_{\omega_{l}} T_{\omega_{l-1}} \cdots T_{\omega_{0}}(x)\right)$ for finite $n$ and 919 we take into account the limits for small $\varepsilon$ and large $n$. 920

Another explanation is that Sauer's result holds for dif- 921 feomorphisms admitting a SRB measure which is singular 922 with respect to Lebesgue and with more than one Lyapunov 923 exponent. In this case we lose the comparison of the densi- 924 ties given by Eq. (A1) and we should evaluate directly the 925 difference $\int_{X} g d \mu_{\varepsilon}-\int_{X} g d \mu$ : this is done, for example, for 926 uniformly hyperbolic attractors in Ref. 30 and for the Hénon 927 attractor in Ref. 15 , but there is no explicit scaling in $\varepsilon$.

928

\section{APPENDIX B: COMPUTATION OF FIDELITY FOR $q x \bmod 1$}

The iterated perturbed map is defined as

$$
T_{\varepsilon}^{n}=q^{n} x+\varepsilon\left(q^{n-1} \xi_{1}+q^{n-2} \xi_{2}+\cdots+q \xi_{n-1}+\xi_{n}\right) \bmod 1 .
$$

The fidelity for functions $\phi, \psi$ which are piecewise $C^{1}$ with 933 a finite number of discontinuities is given by

$$
\begin{aligned}
F_{n}^{\varepsilon} & =\sum_{k, k^{\prime}} \phi_{k} \psi_{k^{\prime}} \int_{0}^{1} e^{2 \pi i q^{n}\left(k-k^{\prime}\right) x} d x \int_{-1}^{1} \frac{d \boldsymbol{\xi}}{2^{n}} e^{2 \pi i k^{\prime} \varepsilon\left(q^{n-1} \xi_{1}+\cdots+\xi_{n}\right)} \\
& =\phi_{0} \psi_{0}+\sum_{|k| \geq 1} \phi_{k} \psi_{-k} S_{n, q}(k \varepsilon)
\end{aligned}
$$

It is trivial to check that the decay of fidelity for any trans- 937 lation on the torus is equivalent to that of the identity $T x=x, 938$ and thus since $S_{n, q}(x) \equiv S^{n}(x)$, Eq. (B1) implies both Eqs. 939 (14) and (17).

940

To estimate $S_{n, q}(x)$ we can use the bound (15) for $S(x), 941$ but we have to distinguish between three regions (we assume 942 $x>0$, in the general case substitute $x$ with $|x|)$ : region 1,943 where $q^{n-1} x<1$, in which we can use, setting $\alpha=\log (2 \pi) \quad 944$

$$
\left|S_{n, q}(x)\right| \leq e^{-\alpha x^{2}\left(1+q^{2}+\cdots q^{2(n-1)}\right)} \leq e^{-\alpha x^{2} q^{2(n-1)}},
$$

region 3 where 946

$$
x q x \cdots q^{n-1} x=x^{n} q^{1+2+\cdots+n-1}=\left(x q^{(n-1) / 2}\right)^{n}>1
$$

or, equivalently, $x q^{(n-1) / 2}>1$, and thus we can use

$$
\left|S_{n, q}(x)\right| \leq\left(2 \pi x q^{(n-1) / 2}\right)^{-n},
$$

and an intermediate region 2 where $x q^{-(n-1) / 2}<1<x q^{n}$. To 950 analyze the behavior of the function in the latter region let us 951 assume that $x q^{m-1}<1<x q^{m}$ with integer $(n-1) / 2<m<n .952$ We can estimate in a different way the terms with $x q^{l}$ greater 953 or smaller than 1 , and thus 


$$
\begin{aligned}
\left|S_{n, q}(x)\right| & \leq \frac{(2 \pi)^{-(n-m)}}{x q^{m} x q^{m-1} \cdots x q^{n-1}} e^{-\alpha x^{2}\left(1+q^{2}+\cdots q^{2(m-1)}\right)} \\
& \leq\left(2 \pi x q^{(n+m-1) / 2}\right)^{-(n-m)} e^{-\alpha x^{2} q^{2(m-1)}} \\
& \leq\left(2 \pi q^{(n-m-1) / 2}\right)^{-(n-m)} e^{-\alpha x^{2} q^{2(m-1)}} .
\end{aligned}
$$$$
956
$$$$
957
$$

958 We can thus use the following bound for $S_{n, q}(x)$ :

959

$$
\left|S_{n, q}(x)\right| \leq\left\{\begin{array}{l}
\exp \left(-\alpha x^{2} q^{2(n-1)}\right) \quad \text { if } x q^{n-1}<1 \\
\left(2 \pi q^{(n-m-1) / 2}\right)^{-(n-m)} e^{-\alpha x^{2} q^{2(m-1)}} \quad \text { if } x q^{m-1}<1<x q^{m} \text { with }(n-1) / 2<m<n \\
\left(2 \pi x q^{(n-1) / 2}\right)^{-n} \quad \text { if } x q^{(n-1) / 2}>1,
\end{array}\right.
$$

960

961

962

963 where we have once again $\alpha=\log (2 \pi)$. [When $q=1$ there is 964 no region 2 , and in region 1 the more accurate bound $S^{n}(x)$ $965 \leq e^{-\alpha x^{2} n}$ can be used.]

966 Using the estimates on $S_{n, q}(x)$ we provide bounds to the 967 fidelity in three different regions. It is convenient to intro968 duce an integer $n_{*}$ defined by $n_{*}=\left\{-\log _{q} \varepsilon\right\}$, where $\{x\}$ de969 notes the integer closest to the real $x$, so that

$970 \varepsilon \simeq q^{-n_{*}}$.

971

972

- Region I: $\varepsilon q^{n-1}<1$. Using the Fourier expansion for the fidelity (B1) we write

$$
\left|F_{n}-\phi_{0} \psi_{0}\right| \leq \sum_{k \neq 0}\left|\phi_{k} \psi_{-k}\right|\left|S_{n, q}(k \varepsilon)\right|=R_{1}+R_{2}+R_{3},
$$

974 where the term $R_{1}$ corresponds to the contribution of 975 all the Fourier components with $|k| \varepsilon q^{n-1}<1$, the term $976 \quad R_{2}$ to the components with $|k| \varepsilon q^{(n-1) / 2}<1<|k| \varepsilon q^{n-1}$, 977 and the term $R_{3}$ to the components with $978 \quad|k| \varepsilon q^{(n-1) / 2}>1$.

979 The following bound holds for the first term $R_{1}$,

$$
\begin{aligned}
R_{1} & \leq \sum_{|k|=1}^{\left(\varepsilon q^{n-1}\right)^{-1}}\left|\phi_{k} \psi_{-k}\right| e^{-\alpha \varepsilon^{2} k^{2} q^{2(n-1)}} \\
& \leq e^{-\alpha \varepsilon^{2} q^{2(n-1)}} 2 \sum_{k=1}^{\left(\varepsilon q^{n-1}\right)^{-1}} \frac{1}{k^{2}} \leq 2\left(2-\varepsilon q^{n-1}\right) e^{-\alpha \varepsilon^{2} q^{2(n-1)},}
\end{aligned}
$$

982 where we have assumed once again that the Fourier coeffi983 cients decay as $|k|^{-1}$ and we have used the following esti984 mate:

$$
\sum_{k=A}^{B} \frac{1}{k^{2}} \leq \frac{1}{A^{2}}+\int_{A}^{B} \frac{d k}{k^{2}}=\frac{1}{A^{2}}+\frac{1}{A}-\frac{1}{B} \leq \frac{2}{A}-\frac{1}{B}
$$

986 for $B>A \geq 1$. The second term $R_{2}$ has the following bound:

$$
\begin{aligned}
R_{2} & \leq \sum_{m=(n+1) / 2}^{n-1}\left(2 \pi q^{(n-m-1) / 2}\right)^{-(n-m)} \sum_{|k|=\left(\varepsilon q^{m}\right)^{-1}}^{|k|=\left(\varepsilon q^{m-1}\right)^{-1}} \frac{e^{-\alpha k^{2} \varepsilon^{2} q^{2(m-1)}}}{k^{2}} \\
& \leq \sum_{m=(n+1) / 2}^{n-1}\left(2 \pi q^{(n-m-1) / 2}\right)^{-(n-m)} e^{-\alpha / q^{2}} 2 \varepsilon q^{m}\left(2-q^{-1}\right) \\
& \leq \frac{e^{-\alpha / q^{2}}}{2 \pi} 2 \varepsilon\left(2-\frac{1}{q}\right) \sum_{m=n+1 / 2}^{n-1} q^{m} \\
& \leq 4 \varepsilon q^{n} \frac{e^{-\alpha / q^{2}}}{2 \pi(q-1)} \leq 2 \varepsilon q^{n-1}
\end{aligned}
$$

For the third term $R_{3}$ we have the bound

$$
\begin{aligned}
R_{3} & \leq \sum_{|k| \geq\left(\varepsilon q^{(n-1) / 2}\right)^{-1}}^{\infty} \frac{1}{k^{2}} \frac{1}{\left(2 \pi \varepsilon|k| q^{(n-1) / 2}\right)^{n}} \\
& \leq \frac{2}{\left(2 \pi \varepsilon q^{(n-1) / 2}\right)^{n}}\left(\left(\varepsilon q^{(n-1) / 2}\right)^{n+2}+\int_{\left(\varepsilon q^{(n-1) / 2}\right)^{-1}}^{\infty} \frac{d k}{k^{n+2}}\right) \\
& =\frac{2}{(2 \pi)^{n}} \varepsilon q^{(n-1) / 2}\left(\frac{1}{n+1}+\varepsilon q^{(n-1) / 2}\right) \\
& \leq \frac{4}{(2 \pi)^{n}} \varepsilon q^{(n-1) / 2}
\end{aligned}
$$

Finally the estimate in this region reads

$$
\begin{aligned}
\left|F_{n}-\phi_{0} \psi_{0}\right| \leq & 2\left(2-\varepsilon q^{n-1}\right) e^{-\alpha \varepsilon^{2} q^{2(n-1)}}+2 \varepsilon q^{n-1} \\
& +\frac{4}{(2 \pi)^{n}} \varepsilon q^{(n-1) / 2}
\end{aligned}
$$

which for $q=1$ leads to Eq. (16).

- Region II: $\varepsilon q^{(n-1) / 2}<1<\varepsilon q^{n-1}$. In this region the estimate 1000 is not very accurate. First of all $R_{1}$ vanishes since the upper 1001 limit in the sum, namely, $\left(\varepsilon q^{n-1}\right)^{-1}$, is smaller than the 1002 lower limit. The second term $R_{2}$, which is evaluated above 1003 for $\varepsilon q^{n-1} \simeq 1$, is of order 1 , whereas the last term $R_{3}$ is 1004 small with respect to 1 .

1005

- Region III: $\varepsilon q^{(n-1) / 2}>1$. In this region the contributions of 1006 $R_{1}$ and $R_{2}$ vanish and we are left just with $R_{3}$ which reads 1007 

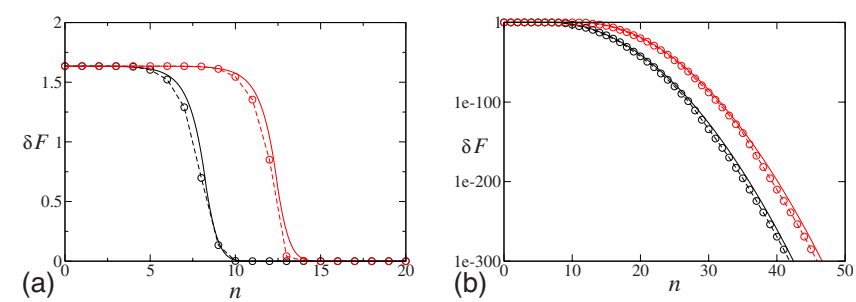

FIG. 21. (Color online) Comparison between the analytical result (B1) (dashed lines and circles) and the estimate (B5) (continuous lines) using $\phi_{k}=\psi_{k}=k^{-1}$ truncated at $k=100$. Black: $\varepsilon=10^{-4}$. Red (gray in black and white): $\varepsilon=10^{-6}$ [(b) logarithmic plot].

1008

$$
\begin{aligned}
R_{3} & \leq \sum_{|k|=1}^{\infty} \frac{1}{k^{2}} \frac{1}{\left(2 \pi \varepsilon|k| q^{(n-1) / 2}\right)^{n}} \\
& =2\left(1+\int_{1}^{\infty} \frac{d k}{k^{n+2}}\right) \frac{1}{\left(2 \pi \varepsilon q^{(n-1) / 2}\right)^{n}} \\
& =\frac{n+2}{n+1} \frac{2}{\left(2 \pi \varepsilon q^{(n-1) / 2}\right)^{n}}
\end{aligned}
$$

\section{1. Fit and comparison to Monte Carlo 1012 simulations}

1013 The validity of the previous estimates has been checked 1014 with numerical calculations. We first write Eq. (B3) using $1015 \varepsilon=q^{-n_{*}}$ so that

1016

$$
R_{3} \leq 3\left(\frac{q^{1 / 2}}{2 \pi}\right)^{n} q^{n n_{*}} q^{-n^{2} / 2}=3 q^{n_{*}^{2} / 2}\left(\frac{q^{1 / 2}}{2 \pi}\right)^{n} q^{-\left(n-n_{*}\right)^{2} / 2},
$$

1017 valid for $\varepsilon q^{(n-1) / 2}>1$. From the estimate (B2) and the present 1018 one we finally obtain

$$
\left|F_{n}-\phi_{0} \psi_{0}\right| \leq\left\{\begin{array}{c}
2\left(2-\varepsilon q^{n-1}\right) e^{-\alpha \varepsilon^{2} q^{2(n-1)}}+2 \varepsilon q^{n-1} \\
\quad \text { if } n<n_{*}+1 \\
3 q^{n_{*}^{2} / 2}\left(\frac{q^{1 / 2}}{2 \pi}\right)^{n} q^{-\left(n-n_{*}\right)^{2} / 2} \\
\text { if } n \geq 2 n_{*}+1 .
\end{array}\right.
$$

1019

1020 We can fit the fidelity with a formula derived from the pre1021 vious estimates by extending the estimate in region III to 1022 region II according to

1023

$$
\left|F_{n}-\phi_{0} \psi_{0}\right| \simeq \begin{cases}A e^{-\alpha q^{2\left(n-n_{*}\right)}} & \text { if } n \leq n_{*} \\ A e^{-\alpha^{\prime}} q^{-\left(n-n_{*}\right)^{2} / 2} & \text { if } n>n_{*}\end{cases}
$$

1024 where $A$ is a constant of order 1 . We can evaluate the width 1025 of the transition $\Delta n$ requiring that at $n=n_{*}-\Delta n$ the decay of 1026 fidelity $\delta F_{n}=\left|F_{n}-\phi_{0} \psi_{0}\right|$ assumes a value some percent below $1027 A$ [at $n=n_{*}$ its value is $\left.A e^{-\alpha}=A(2 \pi)^{-1}=0.16 A\right]$. Choosing, 1028 for instance, $\delta F_{n_{*}-\Delta n}=A e^{\alpha e^{-4}} \simeq 0.97 A$ we find

$1029 \Delta n=\frac{2}{\log q}$.

1030 The validity of the analytical result (B1) has been checked 1031 with a Monte Carlo simulation (Fig. 1). We found (Fig. 21) that the analytical result (B1) can be fitted very well by 1032 Eq. (B5).

1033

We outline that due to the extremely quick decay of the 1034 $e^{-\alpha \varepsilon^{2} q^{2(n-1)}}$ term around $n_{*}$, the fit (B5) can be sketched as a 1035 plateau of length $n_{*}(\varepsilon)$ followed by an $\varepsilon$ independent decay 1036 law.

1037

\section{APPENDIX C: THE CONTINUUM LIMIT}

1038

A map on the torus $\mathbb{T}$ with a random perturbation

1039

$$
x_{n+1}=x_{n}+\Delta t \phi\left(x_{n}\right)+\varepsilon \sqrt{\delta t} \xi_{n},
$$

where $\xi_{n}$ are random variables with zero average and unit 1041 variance, becomes in the limit $\Delta t \rightarrow 0$ the Langevin equation 1042

$$
\dot{x}=\phi(x)+\varepsilon \xi(t),
$$

1043

setting $\xi(t) d t=d w(t)$ where $w(t)$ is a Wiener process and $\xi(t) 1044$ a distribution known as white noise. The probability density 1045 distribution $\rho(x, t)$ satisfies the Fokker-Planck equation

1046

$$
\frac{\partial \rho}{\partial t}+\phi(x) \rho=\frac{\varepsilon^{2}}{2} \frac{\partial^{2} \rho}{\partial x^{2}}
$$

with boundary conditions $\rho(0, t)=\rho(1, t)$ and initial condition 1048 $\rho(x, 0)=\rho_{0}(x)$. The fundamental solution $G\left(x, t ; x_{0}, 0\right)$ satis- 1049 fies the same equation with initial condition $G\left(x, 0 ; x_{0}, 0\right) 1050$ $=\delta\left(x-x_{0}\right)$. As a consequence, we have

1051

$$
\rho(x, t)=\int_{0}^{1} d x_{0} G\left(x, t ; x_{0}, 0\right) \rho_{0}\left(x_{0}\right)
$$

Letting $G_{\mathbb{R}}$ be the fundamental solution defined in $\mathbb{R}$ the 1053 corresponding solution for the torus $\mathrm{T}$ is given by

1054

$$
G_{\epsilon}\left(x, t ; x_{0}, t\right)=\sum_{n=-\infty}^{+\infty} G_{\mathrm{R}}\left(x+n, t ; x_{0}, 0\right)=\sum_{k=-\infty}^{+\infty} C_{k}(t) e^{2 \pi i k x} .
$$

Simple calculations show that for $\phi(x)=\Omega$ and $\phi(x)=A x$ the 1056 Fourier coefficients are given by

1057

$$
\begin{aligned}
& C_{k}(t)=e^{-2 \pi i k\left(x_{0}+\Omega t\right)} e^{-2 \pi^{2} k^{2} \epsilon^{2} t} \quad \text { if } \phi=\Omega, \\
& C_{k}(t)=e^{-2 \pi i k x_{0} e^{A t}} e^{-2 \pi^{2} k^{2} \epsilon^{2}\left(e^{2 A t}-1\right) / 2 A} \quad \text { if } \phi=A x .
\end{aligned}
$$

We notice that the unperturbed $\epsilon=0$ fundamental solution is 1060 $G_{0}\left(x, t ; x_{0}, t\right)=\delta\left(x-S_{t}\left(x_{0}\right)\right)$, where $S_{t}\left(x_{0}\right)=x_{0}+\Omega t$ if $\phi=\Omega$ and 1061 $S_{t}\left(x_{0}\right)=x_{0} e^{A t}$ if $\phi=A x$.

1062

Given two smooth observables $f(x), g(x)$ the correla- 1063 tions and the fidelity are defined by

1064

$$
\begin{aligned}
& C(t)=\int_{0}^{1} d x_{0} f\left(x_{0}\right) \int_{0}^{1} d x G\left(x, t ; x_{0}, 0\right) g(x), \\
& F(t)=\int_{0}^{1} d x_{0} f\left(S_{t}\left(x_{0}\right)\right) \int_{0}^{1} d x G\left(x, t ; x_{0}, 0\right) g(x) .
\end{aligned}
$$


1068

$$
F(t)=\sum_{k=-\infty}^{+\infty} \sum_{k^{\prime}=-\infty}^{+\infty} f_{-k} e^{-2 \pi^{2} k^{\prime 2} \sigma^{2}(t)} g_{k^{\prime}} \int_{0}^{1} d x_{0} e^{-2 \pi i S_{t}\left(x_{0}\right)\left(k-k^{\prime}\right)},
$$

1069 the mean square deviation of the stochastic processes satis1070 fying $\dot{\eta}=\Omega+\epsilon \xi$ is $\sigma^{2}(t)=\epsilon^{2} t$, whereas for $\dot{\eta}=A x+\epsilon \xi$ it is 1071 given by $\sigma^{2}(t)=\epsilon^{2}\left(e^{2 A t}-1\right) / 2 A$. The last integral is $\delta_{k^{\prime}, k}$ if $1072 S_{t}\left(x_{0}\right)=x_{0}+\Omega t$ and $e^{i \theta} \theta^{-1} \sin \theta$ with $\theta=\pi e^{A t}\left(k^{\prime}-k\right)$, which 1073 approaches $\delta_{k^{\prime}, k}$ as $t>0$ grows. As a consequence we may 1074 compare the results with the translations on the torus and the 1075 map $q x$ mod 1 by setting $\omega=\Omega \Delta t, q=1+A \Delta t$, and $\epsilon_{\text {map }}$ $1076=\epsilon \sqrt{\Delta t}$. Agreement is found with the fidelity for the maps if 1077 we let $\Delta t \rightarrow 0$ and $n \rightarrow \infty$ so that $n \Delta t=t$ finite.

\section{APPENDIX D: LÉVY’S INVERSION FORMULA}

1079 Let us consider a 1D map on the unit interval $[0,1]$ and 1080 the observable identity $f(x)=x$. Suppose, moreover, that $\mu$ 1081 and $\mu_{\varepsilon}$ have absolutely continuous invariant densities $h$ and $1082 h_{\varepsilon}$, respectively, which are $\mathcal{L}_{m}^{1}$ very close in such a way that $1083 \varphi_{\infty}^{\varepsilon}$ is approximately given by $\varphi_{\infty}^{\varepsilon}(u)=\left|\int_{[0,1]} e^{i u x} h(x) d x\right|^{2}$. Stan1084 dard results on Fourier series immediately imply that $\varphi_{\infty}^{\varepsilon}$ is 1085 uniformly continuous on $R$, bounded and vanishing at infin1086 ity (Riemann-Lebesgue). We now consider the interesting 1087 cases given by the intermittent maps of Sec. IV C and the 1088 quadratic maps of Sec. IV D for which the invariant density 1089 behaves like $h(x) \sim x^{-\alpha}$, with $0<\alpha<1$ for the intermittent 1090 maps and $\alpha=0.5$ for the particular quadratic map with $a=2$, 1091 and whenever $x$ is close to 0 (for the quadratic map a change 1092 in variable brings the singularity from \pm 1 to zero). We have, 1093 therefore, to investigate the asymptotic behavior for large $|u|$ 1094 of the integral

$1095 \varphi_{\infty}^{\varepsilon}(u)=\left|\int_{[0,1]} \frac{e^{i u x} r(x)}{x^{\alpha}} d x\right|^{2}, \quad 0<\alpha<1$,

1096 where $r$ is a bounded smooth function in the interval $[0,1]$. 1097 Now we have (Ref. 31, p. 519)

$1098\left|\int_{[0,1]} \frac{e^{i u x} r(x)}{x^{\alpha}} d x\right|=\mathcal{O}\left(|u|^{\alpha-1}\right), \quad|u|$ large.

1099 We could therefore conclude that whenever $\alpha<1 / 2$ the char1100 acteristic function $\varphi_{\infty}^{\varepsilon}$ is summable and we can apply the 1101 Lévy inversion formula to get the density of the distribution 1102 function of the asymptotic error. Instead when $1 / 2 \leq \alpha<1$ 1103 we should use a generalization of Eq. (11); if we call $\Phi_{\infty}^{\varepsilon}$ the 1104 distribution function of $\Delta_{\infty}^{\varepsilon}$ and if $-\infty<a<b<\infty$ are conti1105 nuity points for $\Phi_{\infty}^{\varepsilon}$, then (Ref. 12, p. 264)

1106

$$
\Phi_{\infty}^{\varepsilon}(b)-\Phi_{\infty}^{\varepsilon}(a)=\lim _{C \rightarrow \infty} \frac{1}{2 \pi} \int_{-C}^{C} \frac{e^{-i t a}-e^{-i t b}}{i t} \varphi_{\infty}^{\varepsilon}(t) d t .
$$

1107 The integral on the right-hand side of the preceding formula 1108 is of course convergent for $0<\alpha<1$ and whenever $|C|$ goes 1109 to infinity. We have nevertheless proceeded to the numerical computation of the improper integral $\rho_{\infty}^{\varepsilon}(t){ }^{1110}$ $=(2 \pi)^{-1} \int_{-\infty}^{\infty} e^{-i u t} \varphi_{\infty}^{\varepsilon}(u) d u \sim(2 \pi)^{-1} \int_{-\infty}^{\infty} e^{-i u t} /\left(u^{2-2 \alpha}\right) d u$, where 1111 $2-2 \alpha \leq 1$ for $1 / 2 \leq \alpha$. We choose for simplicity $2-2 \alpha<1,1112$ since the integrand $u^{2 \alpha-2}$ is the asymptotic approximation, 1113 for large $|u|$, of the characteristic function $\varphi_{\infty}^{\varepsilon}(u)$, and this one 1114 is surely integrable in the neighborhood of zero. It is well 1115 known that the Fourier transform of $u^{2 \alpha-2}$ and for $2-2 \alpha 1116$ $<1$ is of order $t^{1-2 \alpha}$ in the distribution sense. ${ }^{32}$ Therefore the 1117 density $\rho_{\infty}^{\varepsilon}(t)$ computed numerically blows up to infinity for $t 1118$ close to zero and we observed this fact for the intermittent 1119 and the quadratic map described above. We believe the same 1120 happens for the singular measure of the Baker map, but we 1121 do not even have a heuristic handling for this.

1122

${ }^{1}$ D. Knuth, The Art of Computer Programming (Addison-Wesley, Reading, 1123 1969), Vol. 2.

${ }^{2}$ A. Katok and B. Hasselblatt, Introduction to the Modern Theory of Dy- 1125 namical Systems (Cambridge University Press, Cambridge, 1995). 1126

${ }^{3}$ S. M. Hammel, J. A. Yorke, and C. Grebogi, J. Complex. 3, 136 (1987). 1127

${ }^{4}$ S. M. Hammel, J. A. Yorke, and C. Grebogi, Bull. Am. Math. Soc. 19, 1128 465 (1988).

1129

${ }^{5}$ C. Grebogi, S. Hammel, J. Yorke, and T. Sauer, Phys. Rev. Lett. 65, 15271130 (1990).

${ }^{6}$ S. N. Chow and K. Palmer, J. Dyn. Differ. Equ. 3, 361 (1991).

${ }^{7}$ E. S. Van Vleck, SIAM J. Sci. Comput. (USA) 16, 1177 (1995). 1133

${ }^{8}$ T. Sauer, C. Grebogi, and J. Yorke, Phys. Rev. Lett. 79, 59 (1997). 1134

${ }^{9}$ C. Liverani, Ph. Marie, and S. Vaienti, J. Stat. Phys. 128, 1079 (2007). 1135

${ }^{10}$ G. Benenti, G. Casati, and G. Velbe, Phys. Rev. E 67, 055202 (2003). 1136

${ }^{11} \mathrm{H}$. Hu, Ph. Marie, and S. Vaienti (unpublished). 1137

${ }^{12}$ Y. S. Chow and H. Teicher, Probability Theory (Springer-Verlag, Berlin, 1138 1978).

${ }^{13}$ T. Sauer, Phys. Rev. E 65, 036220 (2002).

1139

${ }^{14}$ M. Benedicks and L. Carleson, Ann. Math. 133, 73 (1991). 1141

${ }^{15}$ M. Benedicks and M. Viana, Ann. Inst. Henri Poincare, Anal. Non Lin- 1142 eaire 23, 713 (2006).

1143

${ }^{16}$ D. Bessis, G. Paladin, G. Turchetti, and S. Vaienti, J. Stat. Phys. 51, 1091144 (1988).

1145

${ }^{17}$ A. Zygmund, Trigonometrical Series, 2nd ed. (Cambridge University 1146 Press, Cambridge, 1968).

1147

${ }^{18}$ D. Bessis, J. D. Fournier, G. Servizi, and G. Turchetti, Phys. Rev. A 36, 1148 920 (1987)

1149

${ }^{19}$ C. Liverani, B. Saussol, and S. Vaienti, Ergod. Theory Dyn. Syst. 19, 6711150 (1999).

${ }^{20}$ L. S. Young, Isr. J. Math. 110, 153 (1999).

1151

${ }^{21}$ H. Hu, Ergod. Theory Dyn. Syst. 24, 495 (2004). 1153

${ }^{22}$ O. Sarig, Invent. Math. 150, 629 (2002). 1154

${ }^{23}$ S. Gouëzel, Isr. J. Math. 139, 29 (2004). 1155

${ }^{24}$ K. K. Lin, Nonlinearity 18, 659 (2005). 1156

${ }^{25}$ V. Araújo and A. Tahzibi, Nonlinearity 18, 939 (2005). 1157

${ }^{26}$ M. Blank, Discrete Contin. Dyn. Syst. 21, 749 (2008). 1158

${ }^{27}$ V. Baladi and M. Viana, Ann. Sci. Ec. Normale Super. 29, 483 (1996). 1159

${ }^{28}$ M. Jakobson, Commun. Math. Phys. 81, 39 (1981). 1160

${ }^{29}$ J. Alves and V. Araujo, Asterisque 286, 25 (2003). 1161

${ }^{30}$ M. Viana, Brazilian Mathematics Colloquium 1997, IMPA. 1162

${ }^{31}$ E. W. Hobson, The Theory of Functions of a Real Variable (Dover, New 1163 York, 1927), Vol. 2.

1164

${ }^{32}$ R. Strichartz, A Guide to Distribution Theory and Fourier Transform 1165 (World Scientific, Singapore, 2003). 


\section{AUTHOR QUERIES — 026904CHA}

\#1 Author, because of the number of changes to your article, please check your corrections carefully and send us an email with your approval or any minor corrections you mays till have. Thank you. 